ARTICLE

https://doi.org/10.1038/s41467-019-09028-v/

\title{
Regnase-1-mediated post-transcriptional regulation is essential for hematopoietic stem and progenitor cell homeostasis
}

Hiroyasu Kidoya (10 1, Fumitaka Muramatsu', Teppei Shimamura², Weizhen Jia1, Takashi Satoh ${ }^{3}$, Yumiko Hayashi ${ }^{1}$, Hisamichi Naito ${ }^{1}$, Yuya Kunisaki ${ }^{4}$, Fumio Arai ${ }^{4}$, Masahide Seki ${ }^{5}$, Yutaka Suzuki ${ }^{5}$, Tsuyoshi Osawa 6 , Shizuo Akira ${ }^{3,7} \&$ Nobuyuki Takakura ${ }^{1}$

The balance between self-renewal and differentiation of hematopoietic stem and progenitor cells (HSPCS) maintains hematopoietic homeostasis, failure of which can lead to hematopoietic disorder. HSPC fate is controlled by signals from the bone marrow niche resulting in alteration of the stem cell transcription network. Regnase-1, a member of the $\mathrm{CCCH}$ zinc finger protein family possessing RNAse activity, mediates post-transcriptional regulatory activity through degradation of target mRNAs. The precise function of Regnase-1 has been explored in inflammation-related cytokine expression but its function in hematopoiesis has not been elucidated. Here, we show that Regnase-1 regulates self-renewal of HSPCs through modulating the stability of Gata2 and Tal1 mRNA. In addition, we found that dysfunction of Regnase-1 leads to the rapid onset of abnormal hematopoiesis. Thus, our data reveal that Regnase-1-mediated post-transcriptional regulation is required for HSPC maintenance and suggest that it represents a leukemia tumor suppressor.

\footnotetext{
${ }^{1}$ Department of Signal Transduction, Research Institute for Microbial Diseases, Osaka University, 3-1 Yamada-oka, Osaka, Suita, 565-0871, Japan. ${ }^{2}$ Division of Systems Biology, Graduate School of Medicine, Nagoya University, 65 Tsurumai-cho, Nagoya, Showa-ku, 466-8550, Japan. ${ }^{3}$ Department of Host Defense, Research Institute for Microbial Diseases, Osaka University, 3-1 Yamada-oka, Osaka, Suita 565-0871, Japan. ${ }^{4}$ Department of Stem Cell Biology and Medicine/Cancer Stem Cell Research, Kyushu University, 3-1-1 Maidashi, Fukuoka, Higashi-ku 812-8582, Japan. ${ }^{5}$ Department of Medical Genome Sciences Graduate School of Frontier Sciences, The University of Tokyo, Chiba 277-8561, Japan. ${ }^{6}$ Division of Integrative Nutriomics and Oncology, Research Center for Advanced Science and Technology, The University of Tokyo, 4-6-1 Komaba, Tokyo, Meguro-ku 153-8904, Japan. ${ }^{7}$ Laboratory of Host Defense, World Premier Institute Immunology Frontier Research Center, Osaka University, Osaka 565-0871, Japan. Correspondence and requests for materials should be addressed to H.K. (email: kidoya@biken.osaka-u.ac.jp) or to N.T. (email: ntakaku@biken.osaka-u.ac.jp)
} 
$\mathrm{T}$ he hematopoietic system is maintained over the lifetime of an organism through the well-orchestrated balance between self-renewal and differentiation of hematopoietic stem and progenitor cells (HSPCs) ${ }^{1}$. The HSPC compartment is heterogeneous and includes long-term hematopoietic stem cells (LT-HSCs) defined by their ability to give rise to all blood cell lineages and sustain life-long self-renewal. The vast majority of LT-HSCs is predominantly quiescent, remaining in the G0 phase of the cell cycle; the change to proliferative $\mathrm{S}+\mathrm{G} 2 / \mathrm{M}$ phase in response to hematological stress is a key event in hematopoietic homeostasis ${ }^{2}$. Quiescent LT-HSCs reside mainly in bone marrow (BM) niches, and their fate is controlled by multiple secreted and cell-surface molecules in the BM microenvironment ${ }^{3}$. Signals from the BM niche control HSPC fate via a variety of signaling pathways and transcription factors. Transcriptional regulation of gene expression through transcription networks plays crucial roles in hematopoiesis and in the maintenance of $\mathrm{HSPCs}^{4}$. Although various key transcription factors involved in HSPC homeostasis have been identified, regulatory mechanisms controlling the transcriptional network regulating hematopoiesis remain undetermined.

HSPCs maintain life-long hematopoiesis by self-renewal, which provides an opportunity for the accumulation of multiple genetic abnormalities. Accumulated chromosomal translocations and gene mutations can lead to malignant transformation of HSPCs and generation of leukemic stem cells (LSCs). It is widely accepted that LSCs acquire aberrant self-renewal capacity in contrast to normal HSPCs which have restricted self-renewal capacity and mostly remain in the quiescent state, ${ }^{5}$ this results in the development of leukemia ${ }^{6}$. LSCs are also thought to be responsible for leukemia maintenance, therapy failure and disease relapse $^{7}$. Acute myeloid leukemia (AML) is the most common type of leukemia in adults, characterized by the uncontrolled proliferation of abnormal and dysfunctional progenitor cells (blasts) in the BM. Transcriptional deregulation through aberrant expression and frequent mutation of transcription factors has been reported in AML patients ${ }^{8}$. Such abnormal transcriptional regulation leads to leukemogenesis and is crucially involved in the pathogenesis of AML.

The efficiency of mRNA translation is strictly controlled by post-transcriptional gene regulation. Cis-acting elements located in the $3^{\prime}$-untranslated region ( $3^{\prime} \mathrm{UTR}$ ) of mRNA plays a key role in the modulation of mRNA stability ${ }^{9,10}$. These elements enable the recognition of target mRNA transcripts by RNA-binding proteins, and promote nuclease-dependent degradation ${ }^{11,12}$. The $\mathrm{CCCH}$ zinc finger protein Regnase-1 encoded by the ZC3H12A (MCPIP1) gene has been identified as a ribonuclease that suppresses gene expression through degradation of transcripts ${ }^{13,14}$. This protein acts as a negative regulator of inflammatory responses by destabilizing inflammation-related cytokines and transcription factor mRNAs such as interleukin-1 $\beta(I L-1 \beta), I L-2$, $I L-6, I L-12 p 40$, and $c-R e l^{13-16}$. A recent study revealed that Regnase-1 binds to target mRNA via reorganization of the conserved stem-loop structure on the $3^{\prime}$ UTR of these genes ${ }^{17}$. In addition to its immune response functions, Regnase-1 is also involved in a wide variety of biological processes such as brain development and adipogenesis by controlling cell differentiation and apoptosis ${ }^{18,19}$.

In the present study, to elucidate cell-intrinsic mechanisms of HSPC homeostasis, we explored which molecules are essential for the regulation of gene expression. Using bioinformatics analysis of gene expression data during HSPC development, the ribonuclease Regnase-1 was identified as a major player. To study essential functions of Regnase-1 in HSPCs, we generated conditional knockout mice in which Regnase-1 was deleted specifically in HSPCs. Here, we document crucial roles of Regnase-1 for self- renewal of HSPCs. In addition, we show the relevance of the lack of Regnase-1 for leukemogenesis, associated with the aberrant expression of key regulators of hematopoiesis.

\section{Results}

Identification of genes important for HSPC maintenance. To identify factors crucial for the determination of HSPC selfrenewal and differentiation, we performed genome-wide gene expression analysis of Lineage $\mathrm{Sca}^{+}{ }^{+} \mathrm{c}-\mathrm{Kit}^{+}$(LSK) HSPCs in different developmental stages using available GEO data sets (GSE69760). The expression of multiple genes was different in HSPCs from adult BM and E14.5 FL. We screened genes encoding intercellular proteins for those highly expressed in adult HSPCs relative to HSPCs from embryos (Fig. 1a). Among these genes, we selected the gene encoding Regnase-1 because this molecule has been reported to associate with mesenchymal stem cell differentiation ${ }^{20}$. The amount of Regnase-1 expression in neonates was greater than in the fetus, and even greater in adults (Fig. 1b). To determine the expression profile of Regnase-1 in HSPC subpopulations, we isolated hematopoietic cells (HC; CD45 ${ }^{+}$), LSK-HSPCs, immature and quiescent $\left(\mathrm{CD} 34^{-}\right.$HSCs; CD34- Flt3- LSK), active (CD34+ ${ }^{+}$HSCs; CD34 ${ }^{+}$Flt3 $^{-}$LSK), and multipotent progenitors (MPPs; $\mathrm{CD} 34^{+} \mathrm{Flt}^{+}$LSK) from adult C57BL/6 WT mice $21-23$. The level of Regnase-1 mRNA was then determined by qRT-PCR. We found that Regnase-1 was relatively highly expressed in all HSPC subsets compared to the whole population of lineage-committed cells and differentiated progenitor cells (Fig. 1c, Supplementary Fig. 1a). Immunohistochemical staining of BM tissue from the femur revealed that Regnase-1 protein was predominantly present in c-Kit-positive cells including HSPCs (Fig. 1d).

Effects of Regnase-1 deletion on BM HSPC populations. To elucidate the function of Regnase-1 in HSPC development and/or maintenance, we analyzed mice harboring a floxed allele of the gene encoding Regnase-1 ${ }^{16}$ mated with mice expressing hematopoietic-specific Cre recombinase under the transcriptional control of vav promoter elements ${ }^{24}$. To confirm the deletion of Regnase-1 in CD34- HSC, we performed qRT-PCR analysis of sorted CD34- HSC from 8-week-old control Reg1 $1^{\text {llox/flox }}$ (Regnase- $\left.1^{\text {flox/flox }}\right), \operatorname{Reg} 1^{\Delta /+}\left(\right.$ Vav1-iCre; Regnase- $\left.1^{\text {flox } /+}\right)$, and Reg1 ${ }^{\Delta / \Delta}$ (Vav1-iCre; Regnase-1 $1^{\text {flox/flox }}$ ) mice. As expected, Regnase-1 expression was almost completely absent in the CD34HSC of Reg1 $1^{\Delta / \Delta}$ and reduced by approximately half in Reg1 $1^{\Delta /+}$ (Fig. 1e). Reg $1^{\Delta / \Delta}$ mice were born at predicted Mendelian ratios, but they exhibited growth reduction compared with their littermate controls. Flow cytometric analysis of HSPC subpopulations in adult $\mathrm{BM}$ revealed that $\operatorname{Reg} 1^{\Delta / \Delta}$ mice possessed higher proportions of LSK-HSPCs and CD34-Flt3- phenotypically defined CD34- HSCs (Fig. 1f, g). Although the total BM cell count was decreased in Reg1 ${ }^{\Delta / \Delta}$ mice relative to littermate controls, the absolute numbers of LSK-HSPCs were significantly increased (Fig. 1h). Furthermore, a marked increment in the absolute number of $\mathrm{CD} 34^{-}$HSCs and CD34 ${ }^{+}$HSC in Reg1 ${ }^{\Delta / \Delta}$ mice was observed (Fig. 1i). Consistent with these results from flow cytometry, histological analysis revealed a significant expansion of

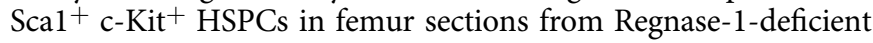
animals (Fig. 1j). In summary, our findings suggest that Regnase1 is widely expressed in HSPCs and contributes to regulation of CD34- HSC proliferation and differentiation.

Loss of Regnase-1 leads to accumulation of immature HSPCs. Definitive hematopoiesis starts in the aorta-gonad-mesonephros (AGM); HSC translocate to the fetal liver (FL) where they undergo expansion and differentiation. Unlike the FL, molecular 


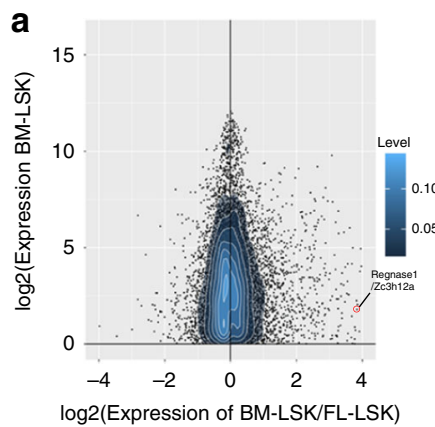

b

\begin{tabular}{lccc}
\hline Symbol & BM HSPC & FL HSPC & Ratio \\
\hline Aldh1a1 & 10.31 & 0.06 & 187.3 \\
Sult1a1 & 2.98 & 0.02 & 166.5 \\
Hk3 & 9.09 & 0.21 & 44.1 \\
Sdsl & 51.19 & 1.81 & 28.3 \\
Galnt6 & 33.75 & 1.28 & 26.3 \\
Zc3h12a & 3.47 & 0.24 & 14.3 \\
Nlrc5 & 4.74 & 0.33 & 14.2 \\
Slc16a11 & 17.87 & 1.39 & 12.9 \\
Padi4 & 17.77 & 1.51 & 11.8 \\
Socs3 & 55.78 & 5.13 & 10.9 \\
\hline
\end{tabular}
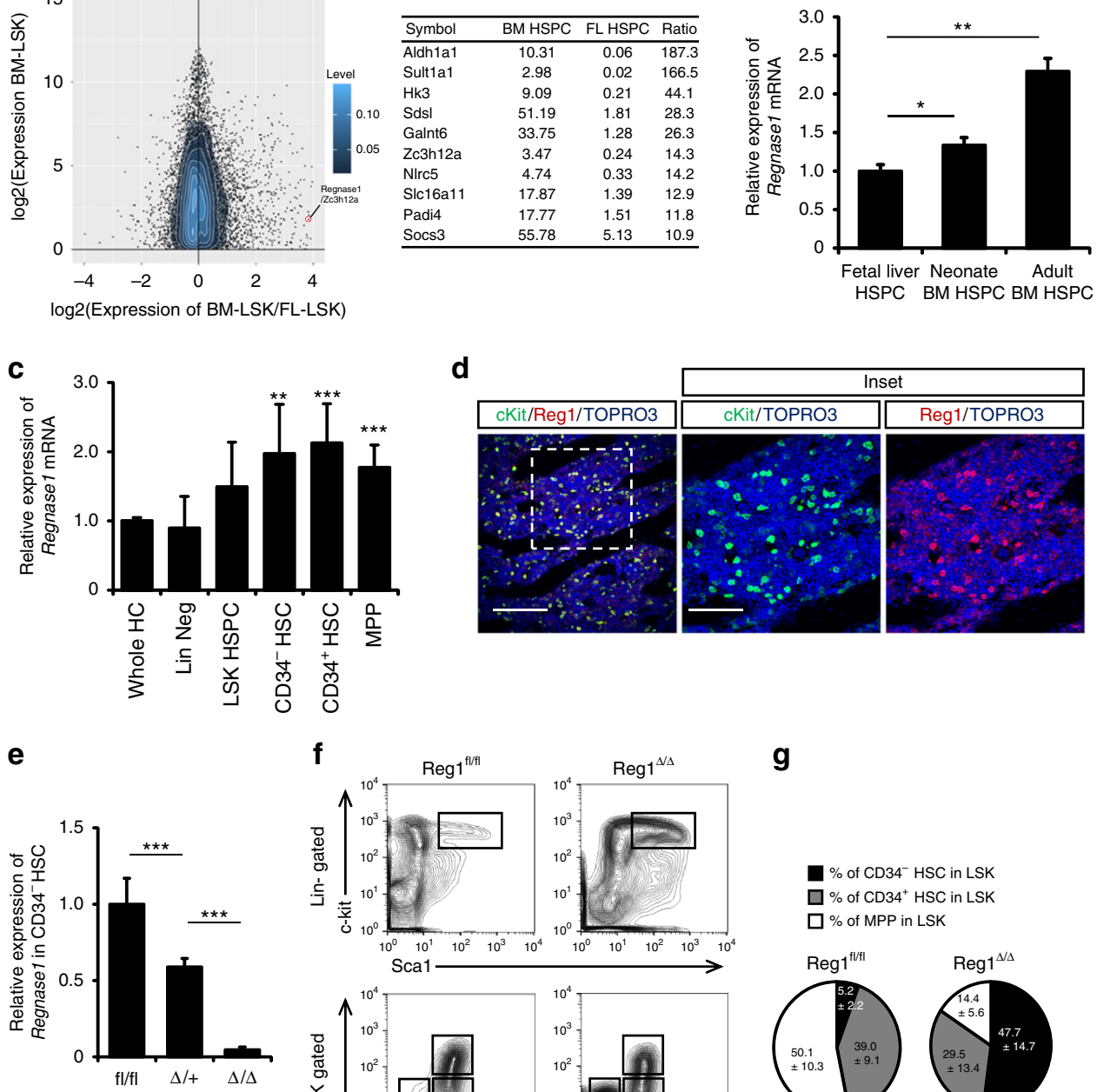

f

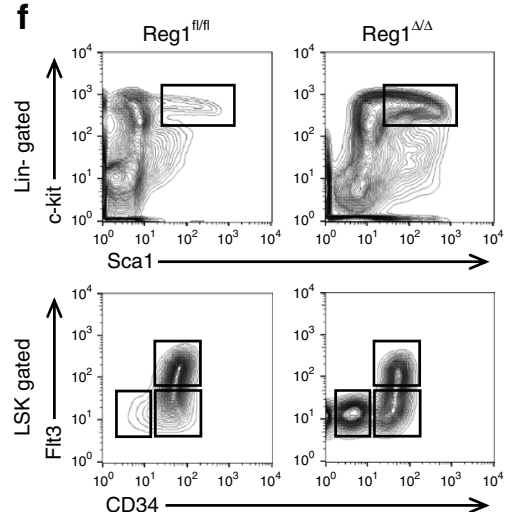

g
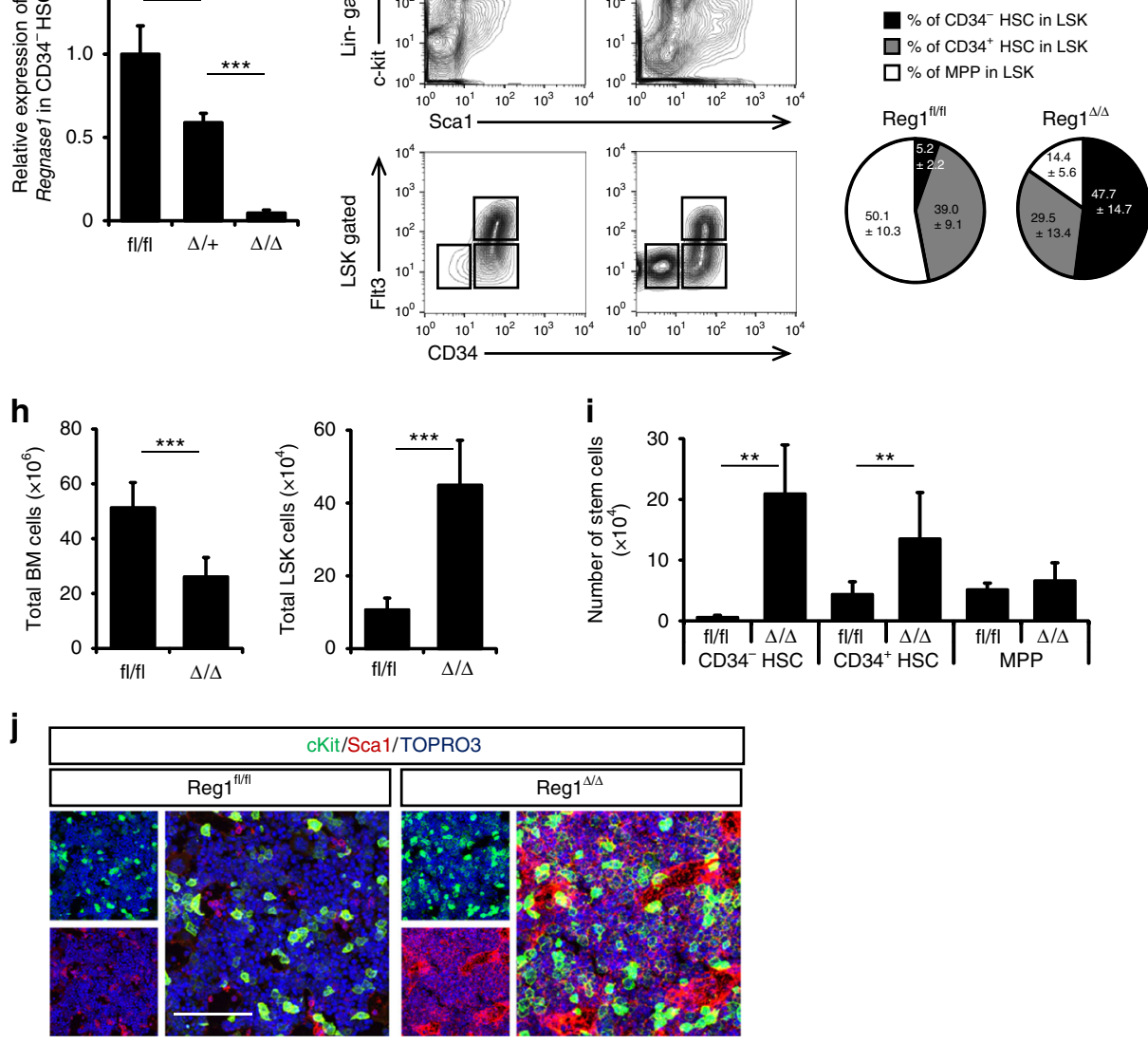

cues from the adult BM microenvironment induce growth arrest of HSCs in the G0 phase of the cell cycle and maintain HSC quiescence ${ }^{25}$. Because Regnase-1 expression is upregulated in HSPCs of adult BM compared with those of the fetus, this suggests the involvement of Regnase-1 in the regulation of the HSC pool. Therefore, we investigated when the anomalous increase of HSPCs occurs in Regnase-1-deficient mice. Flow cytometric analysis of FL cells from E15.5 embryos and BM cells from 1-dayold neonates revealed no differences in the numbers or proportions of HSPCs. However, we found abnormal accumulations of 
Fig. 1 Regnase- 1 is expressed in HSPCs and is involved in maintenance of the HSC pool. a Mean difference plot of mRNA expression in Lineage ${ }^{-}$Sca-1+ ${ }^{-}$ $\mathrm{Kit}^{+}$(LSK) HSPCs from adult BM and HSPCs from E14.5 FL using the GEO data sets (GSE69760). Genes with expression levels increased $\geq 10-$-fold in adult BM and encoding intra-cellar proteins are listed in the table on the right. $\mathbf{b}$ Quantitative RT-PCR of Regnase-1 expression in isolated LSK cells from E14.5 fetal liver, neonate BM, and adult BM ( $n=3$ per group). Data are expressed as fold-change relative to fetal liver HSPCs. c Regnase-1 mRNA expression in lineage-negative cells (Lin Neg), Lin ${ }^{-} \mathrm{cKit}^{+}$Sca-1+ ${ }^{+}$cells (LSK HSC), LSK CD34- Flt3- (CD34- HSC), LSK CD34+ Flt3 $^{-}$(CD34+ $^{+}$HSC), and LSK CD34 Flt3+ (MPP) prepared from BM of adult mice (8-week-old) $(n=3)$. d Immunohistochemical staining of Regnase- 1 in BM. The right-hand panels show a higher magnification of the areas indicated by the boxes. The scale bars represent $200 \mu \mathrm{m}$ and $100 \mu \mathrm{m}$ (insets). e Loss of Regnase- 1 expression in CD34-

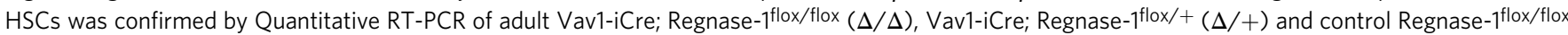
$(\mathrm{fl} / \mathrm{fl})$. The level of Regnase-1 mRNA of the $\mathrm{fl} / \mathrm{fl}$ mice was set at $1.0(n=3$ mice of each genotype). $\mathbf{f}-\mathbf{i}$ Representative flow cytometric analysis $(\mathbf{f})$, quantitative and statistical analyses of HSPCs populations $(\mathbf{g})$, and the numbers $(\mathbf{h}, \mathbf{i})$ in the BM of 8-week-old control (fl/fl) and Regnase-1-KO ( $\Delta / \Delta)$ mice $\left(n=10\right.$ per group). $\mathbf{j}$ Immunohistochemical staining of Sca- $1^{+}$c-Kit ${ }^{+}$HSPCs in control and Regnase-1-KO $(\Delta / \Delta)$ BM. The scale bars represent 100 $\mu \mathrm{m}$. Error bars indicate mean $\pm \mathrm{SD}$. ${ }^{\star} p<0.05 ;{ }^{\star \star} p<0.01,{ }^{\star \star \star} p<0.005$, Tukey-Kramer multiple comparison test in $\mathbf{b}, \mathbf{c}, t$-test in $\mathbf{c}, \mathbf{h}, \mathbf{i}$

LSK-HSPCs and CD34- HSCs in 2-week-old Reg1 ${ }^{\Delta / \Delta}$ mice (Fig. 2a, b). This suggests an inhibitory role for Regnase-1 in HSPC proliferation and that it could contribute to the maintenance of $\mathrm{CD} 34^{-}$HSC quiescence. We investigated the activity of Regnase-1-deficient CD34- HSC by analyzing the cell surface markers CD150 and CD48, useful for determining HSC quiescence; ${ }^{26}$ quiescent HSC subsets are defined as the CD150+ $\mathrm{CD}^{-} 8^{-}$LSK population. We determined the frequency and number of quiescent HSCs in Regnase-1-deficient mice, but found no obvious changes compared with HSCs from WT mice $\left(\operatorname{Reg} 1^{\mathrm{fl} / \mathrm{fl}}\right)$. On the other hand, the frequencies and numbers of CD $150^{+}{ }^{-C D} 48^{+}$LSK-HSPCs and CD150-CD $48^{+}$LSK-HSPCs were significantly increased, and these cells were actively cycling (Fig. 2c, d, Supplementary Fig. 1b, c). Generally, CD150 ${ }^{+} \mathrm{CD} 48^{+}$ LSK-HSPCs are defined as differentiating HSPCs ${ }^{26}$. However, CD48 expression on HSCs was also reported as a marker for cell cycle activation ${ }^{2,27,28}$, and CD $150^{+} \mathrm{CD} 48^{+}$LSK cells include HSCs with colony-forming ability and long-term multi-lineage reconstitution potential similar to LSK-SLAM cells ${ }^{29,30}$. Moreover, the increased $\mathrm{CD} 150^{+} \mathrm{CD} 48^{+}$LSK population in $\operatorname{Reg} 1^{\Delta / \Delta}$ mice did not express the CD34 marker of differentiated HSC, suggesting that Regnase- 1 deficiency resulted in increase of activated CD34HSCs in BM.

CD34- $^{-}$HSC function is impaired by deletion of Regnase-1. We next examined whether increased LSK-HSPCs and CD34- HSCs in $\operatorname{Reg} 1^{\Delta / \Delta}$ retain the ability to undergo normal hematopoietic differentiation in an in vitro CFC assay. LSK-HSPCs from $\operatorname{Reg} 1^{\Delta / \Delta}$ mice exhibited no defects in colony formation and frequencies of different types of colonies compared with their littermate controls (Fig. 2e). In contrast, CD34- HSCs from $\operatorname{Reg} 1^{\Delta / \Delta}$ mice demonstrated an approximately 2 -fold decrease in colony number with slight effect on frequencies of colony types (Fig. 2e). These data indicate that Regnase-1 is involved in the differentiation of CD34- HSCs. To test the quality of Regnase-1deficient CD34- HSCs in vivo, we performed competitive BM transplantation (BMT) assays. Sorted CD34- Flt3- LSKs from the $\mathrm{BM}$ of $\operatorname{Reg} 1^{\Delta / \Delta}$ or control mice $\left(\mathrm{CD} 45.2^{+}\right)$mixed with congenic $\left(\mathrm{CD} 45.1^{+}\right) \mathrm{WT}$ BM cells were transferred into lethally (10 Gy)irradiated WT $\left(\mathrm{CD} 45.1^{+}\right)$mice. Recipient $\mathrm{BM}$ and peripheral blood (PB) cells were then analyzed by flow cytometry 16 weeks

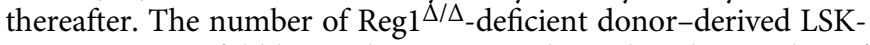
HSPCs was 3.4-fold lower than in control BM, but the number of CD34- HSCs from Reg1 ${ }^{\Delta / \Delta_{\text {}}}$-deficient donors was 3.2-fold higher than in controls (Fig. 2f, g). These data suggest a diminished contribution of Reg1 $1^{\Delta / \Delta}$ CD $34^{-}$HSCs to CD $34^{+}$HSCs and MMPs relative to control cells under competitive conditions, at the same time as the proliferative activity of $\operatorname{Reg} 1^{\Delta / \Delta} \mathrm{CD} 34^{-}$ HSCs is increased.

Next, to investigate the long-term repopulating potential of $\operatorname{Reg} 1^{\Delta / \Delta}$ CD34- HSCs, we analyzed donor cell reconstitution in the peripheral B lymphoid (B220), $\mathrm{T}$ lymphoid (CD3), and myeloid (CD11b) populations of recipient mice. Although $\operatorname{Reg} 1^{\Delta / \Delta}$ animals retained the ability to produce both myeloid and lymphoid lineages, $\operatorname{Reg} 1^{\Delta / \Delta}$ CD34- HSCs contributed to only $16.3 \%$ of B cells, $20.5 \%$ of T cells, and $10.5 \%$ of myeloid cells. This was a markedly lower contribution compared with dominant effects of HC lineage cells from control CD34- HSCs (Fig. 2h, i). By analyzing earlier time points one month after transplantation, we found increased CD34 ${ }^{+}$LSK cells, MEPs and GMPs in the group transplanted with CD34- HSCs from Regnase-1-deficient mice (Supplementary Fig. 1d). This suggests that Regnase-1 deficiency results in abnormal regulation of cell growth and induces excessive proliferation.

Together, these results indicate that Regnase-1-deficient CD34- HSCs engraft normally, but their repopulation potential is significantly attenuated relative to control, consistent with the results from the in vitro colony forming assays. In summary, these data suggest that Regnase-1 plays an important role in regulating the balance between proliferation and differentiation of CD $34^{-}$HSCs.

Regnase-1 regulates HSPC cell cycle activity. Results from Regnase-1-deficient mice suggest that the balance between quiescence and proliferative status of CD34- HSC is regulated by this enzyme. To identify the genes differentially expressed between control and Reg ${ }^{\Delta / \Delta}$ CD $34^{-}$HSCs, global gene expression profiling was performed. Gene ontology (GO) terms related to cell cycle regulation and hematopoietic differentiation were enriched in Reg1 ${ }^{\Delta / \Delta}$ CD34- HSCs (Fig. 2j). Based on this result, we assessed whether the activity of Regnase-1 in HSPC maintenance was by means of regulating the cell cycle and/or apoptosis. To evaluate the cell cycle status of $\mathrm{CD}^{-}{ }^{-} \mathrm{HSC}$ and CD $34^{+}$HSC from Reg1 ${ }^{\Delta / \Delta}$ mice, cells were labeled with EdU and 7-AAAD and analyzed for G0/G1, G2, and G2/M populations. As expected, Regnase-1 deletion led to an increase in the number of cells in all cell cycle phases in both the CD34+ ${ }^{+}$SSC and CD34HSC fractions, and an especially notable increase in cell number in S phase CD34- HSCs (Fig. 3a, b). In contrast, there was only a slight effect on the cell cycle of MPPs and lineage-committed cells (Supplementary Fig. 1e). These results indicate that deletion of Regnase-1 in HSPCs mainly affects cycling of CD34- HSCs and CD $34^{+}$HSCs. Consistent with this, immunohistochemistry confirmed that BrdU incorporation was also increased in c-Kitpositive HSPCs from Reg1 ${ }^{\Delta / \Delta}$ BM (Fig. 3c, d). Next, we performed Pyronin Y/Hoechst 33342 staining to distinguish between cells in the quiescent G0 phase and the proliferating G1-phase. Flow cytometric analysis revealed a marked increase in the number of cells at all phases of the cell cycle, but the proportion of proliferating cells in G1 and S/G2/M (Pyronin $\mathrm{Y}^{+}$Hoechst $^{-}$ and Pyronin $\mathrm{Y}^{+}$Hoechst $^{+}$) was increased relative to quiescent cells at G0 (Pyronin $\mathrm{Y}^{-}$Hoechst $^{-}$) in $\mathrm{CD}^{+} 4^{+}$HSCs from 
a
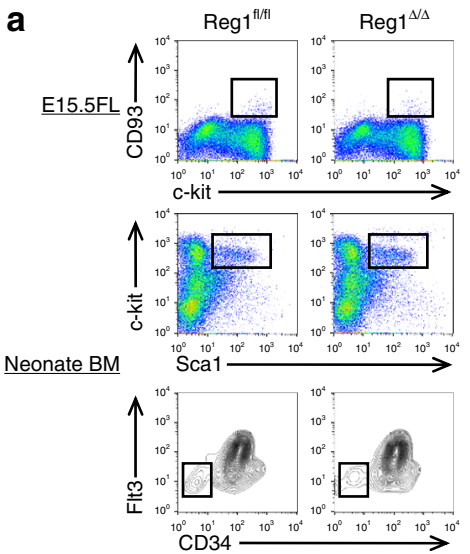

2 weeks BM

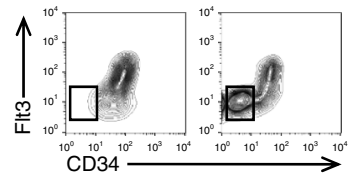

f
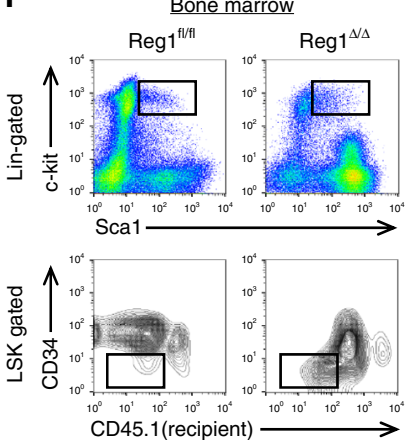

g

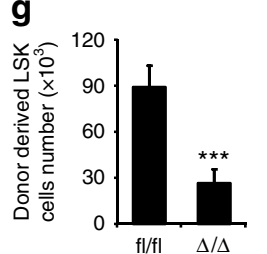

j
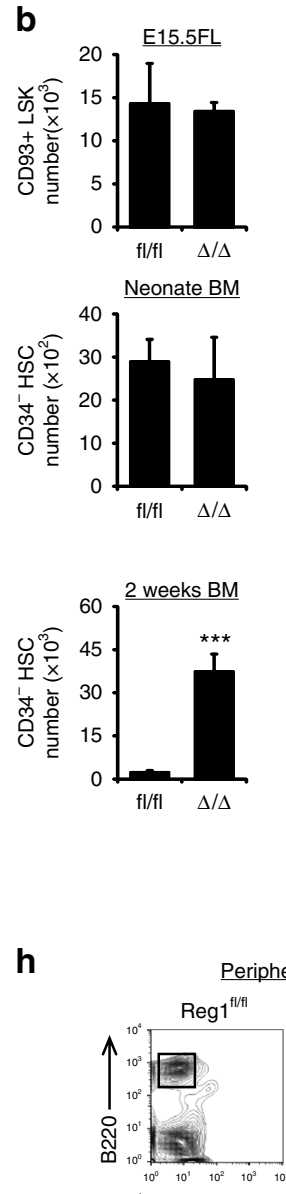

Perip
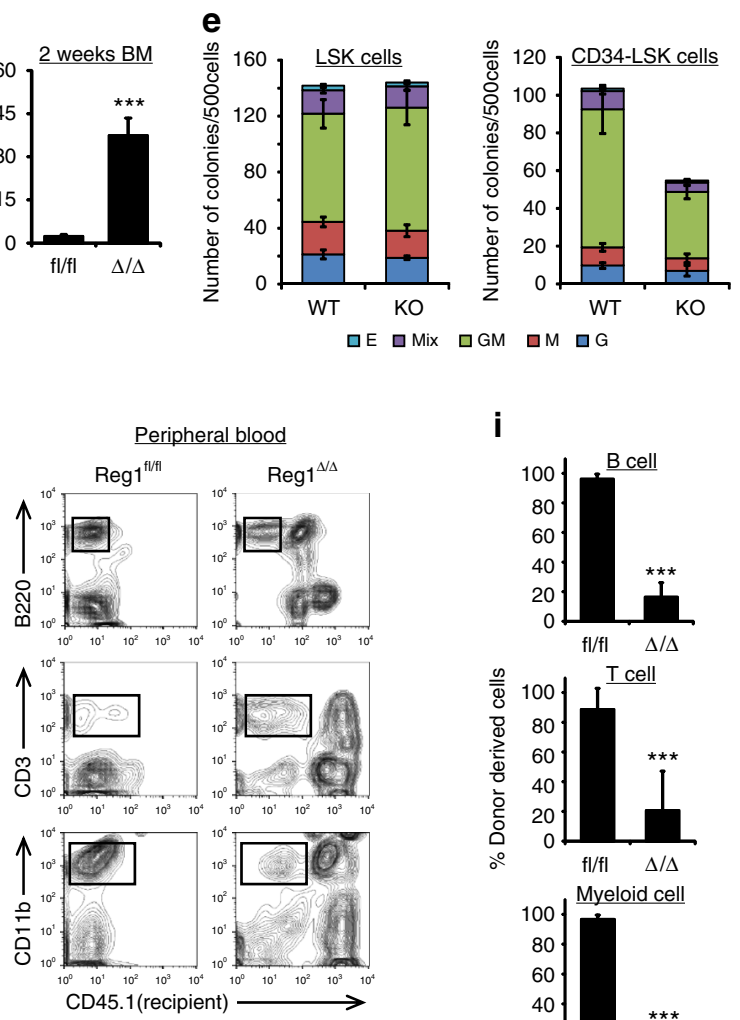

i
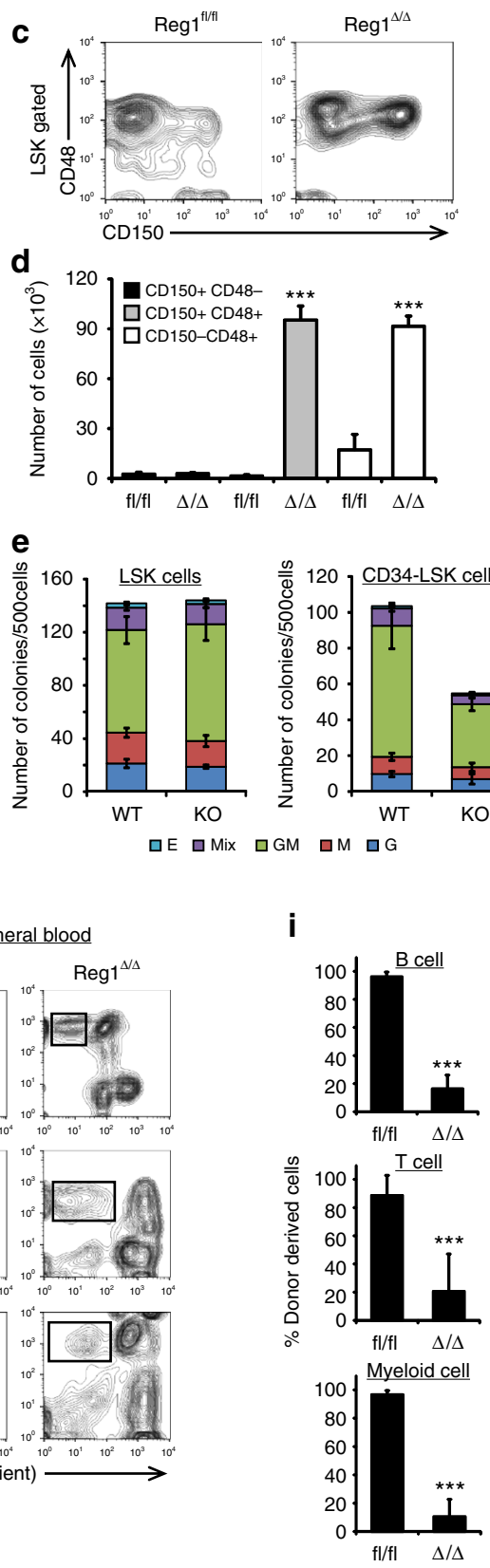

d

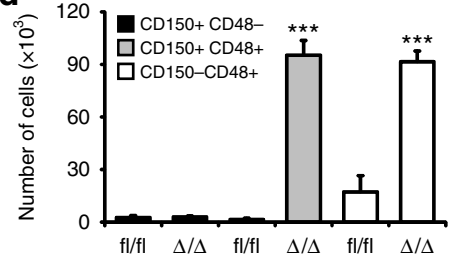


Fig. 2 Regnase-1 deficiency leads to expansion of immature HSPCs. a Representative flow cytometric analysis of HSPC populations in the FL of E15.5 embryo, BM of 1-day-old neonate, BM of 2-week-old control ( $\mathrm{fl} / \mathrm{fl})$ or Regnase-1-KO $(\Delta / \Delta)$ mice $(n=3$ per group). b The numbers of LSK-HSCs or CD34HSCs in the total FL or BM cells assessed by flow cytometry. c Representative flow cytometric analysis of LSK cells for CD48 and CD150 expression of 8 week-old control $(\mathrm{fl} / \mathrm{fl})$ or Regnase-1-KO $(\Delta / \Delta)\left(n=3\right.$ per group). d Percentage of CD150+ CD48- cells, CD150 ${ }^{+} \mathrm{CD}^{-} 8^{+}$cells, and CD150- CD48 ${ }^{+}$cells of control $(\mathrm{fl} / \mathrm{fl})$ and Regnase-1-KO $(\Delta / \Delta)$ mice. e Colony-forming ability of LSK-HSCs and CD34- HSC from control (fl/fl) or Regnase-1-KO $(\Delta / \Delta)$ mice BM. G CFU-granulocyte; GM CFU-granulocyte/monocyte; M CFU-monocyte; E CFU-erythroid; and mix mixed CFU-granulocytes, monocyte, erythroid, and megakaryocyte. Data show the means \pm SD ( $n=5 ; 3$ independent experiments). f Competitive repopulation assay of lethally irradiated (10 Gy) WT recipients (CD45.1) transplanted with $\mathrm{BM}$ of either control $(\mathrm{fl} / \mathrm{fl})$ or Regnase-1-KO $(\Delta / \Delta)$ mice. Chimerism was analyzed in recipient mice 16 weeks after injection of $1 \times 10^{3}$ of CD34- HSCs from control or Regnase-1-KO mice (CD45.2) with $5 \times 10^{5}$ competitor BM cells (CD45.1). Donor-derived cell ratios $\left(C D 45.1^{-}\right)$in BM HSPC and CD34- HSC populations were determined by flow cytometry ( $n=5$ per group). $\mathbf{g}$ The number of LSK or CD34- LSK cells in the BM of mice 16 weeks after transplantation. $\mathbf{h}$ Hematopoietic engraftment of transplanted HSPCs for reconstitution of peripheral blood was analyzed by flow cytometry at 16 weeks after transplantation ( $n=5$ per group). $\mathbf{i}$ The contribution of transplanted donor-derived cells (CD45.1 ${ }^{-}$) to B-cells (B220 ${ }^{+}$), Tcells $\left(\mathrm{CD}^{+}\right)$, and myeloid cells $\left(\mathrm{CD} 11 \mathrm{~b}^{+}\right)$from control $(\mathrm{fl} / \mathrm{fl})$ or Regnase-1-KO $(\Delta / \Delta)$ HSPCs are shown. Data summated from 3 independent experiments and an average of 6 recipient mice in each group with SD. $\mathbf{j}$ Gene ontology (GO) analysis of genes upregulated in the CD34- $4^{-} \mathrm{CS}_{\mathrm{S}}$ from Regnase-1-KO BM based on the GO biological process annotations. Error bars indicate mean $\pm \mathrm{SD}$. ${ }^{\star} p<0.05 ;{ }^{\star \star} p<0.01,{ }^{\star \star \star} p<0.005$, two-sided $t$-test in $\mathbf{b}, \mathbf{d}, \mathbf{g}, \mathbf{i}$

BM was due to cell cycle progression, and that apoptosis was not responsible.

Next, to elucidate the function of Regnase-1 following hematopoietic stress, we subjected $\operatorname{Reg} 1^{\Delta / \Delta}$ mice to myelotoxic stress using 5 -FU. Reg1 ${ }^{\Delta / \Delta}$ mice were more sensitive to repetitive 5 -FU administration, and died significantly earlier than control Reg $1^{\text {flox/flox }}$ mice (Fig. 3j). To evaluate the impact of Regnase-1 deletion during BM ablation and recovery, the kinetics of HSC repopulation were established after a single injection of 5-FU. Consistent with results under the steady state, LSK-HSCs from $\operatorname{Reg} 1^{\Delta / \Delta}$ mouse BM rapidly increased more than in control LSKHSCs after 5-FU treatment. The absolute numbers of LSK-HSCs on day 4 after 5-FU treatment also significantly increased in the $\mathrm{BM}$ of Reg $1^{\Delta / \Delta}$ mice relative to controls (Fig. 3k, 1). 5-FU induces apoptosis of proliferating cells, but many quiescent G0 phase LSK-HSCs in Regnase-1-deficient mice show resistance to 5-FU and may lead to subsequent increase. After 5-FU treatment, Regnase-1 expression in LSK-HSCs from WT mice was upregulated, coinciding with HSPC expansion (Fig. $3 \mathrm{~m}$ ). These data demonstrate that Regnase- 1 has a critical role in the suppression of cell cycle entry and thereby, Reg1 deficiency induces excessive activation and proliferation of HSPCs after myeloablative stress.

Regnase-1 deficiency causes impaired hematopoietic function. Because Regnase- 1 deficiency results in an excessive expansion of HSPCs and a decline in the maintenance of the hematopoietic system, we investigated whether Regnase-1 deletion leads to overt disease. We followed the long-term survival and changes in hematopoiesis of $\operatorname{Reg} 1^{\Delta / \Delta}$ mice. Strikingly, all such mice gradually developed abnormal hematopoiesis, and lost weight, and died within 110 days (Fig. 4a). Macroscopically, 10-week-old $\operatorname{Reg} 1^{\Delta / \Delta}$ mice exhibited severe splenomegaly and mesenteric lymphadenopathy (Fig. 4b, c). Marked decreases of red blood cells, platelets, and hemoglobin concentration, and slight or no alteration of erythropoiesis and megakaryopoiesis were observed in $\operatorname{Reg} 1^{\Delta / \Delta}$ mice (Supplementary Fig. 2a-e). PB of these 8 week-old mice had an elevated granulocyte population $\left(\mathrm{Gr}^{+}\right)$and reduced $\mathrm{T}$ lymphoid population $\left(\mathrm{CD}^{+}\right)$relative to control Reg $1^{\text {flox/flox }}$ animals (Fig. 4d, e). Reg1 ${ }^{\Delta / \Delta}$ BM also contained significantly expanded $\mathrm{Grl}^{+}$and $\mathrm{Macl}^{+}$myeloid populations and reduced lymphoid populations (Fig. 4f, g). Juvenile Reg $1^{\Delta / \Delta}$ mice (2-weeks-old) also exhibited moderate abnormalities in lineage-committed cells in the PB and BM (Supplementary Fig. 3a, b). On the other hand, hematopoietic-specific Regnase-1 hetero-knockout Reg1 ${ }^{\Delta /+}$ mice (Vav1-iCre; Regnase-1 $1^{\text {flox/+}}$ ) were viable, and showed normal growth and appearance until they were 6-months-old. They then showed weakness later, and almost all died within 14 months (Fig. $4 \mathrm{~h})$. Reg $1^{\Delta /+}$ mice also displayed severe splenomegaly and mesenteric lymphadenopathy (Fig. 4i, j), and expansion of LSKHSPC and CD34- HSC subpopulations in BM (Fig. 4k, l). Flow cytometric analysis of $\mathrm{PB}$ and $\mathrm{BM}$ cells of the $\operatorname{Reg} 1^{\Delta /+}$ mice revealed a tendency for increased myeloid populations and decreased lymphoid populations, similar to $\operatorname{Reg1}^{\Delta / \Delta}$ mice (Fig. $4 \mathrm{~m}$ ). This suggests that a single Regnase-1 allele is insufficient for maintaining normal hematopoiesis. Collectively, these results imply an indispensable role for Regnase-1 in the maintenance of normal hematopoiesis and that a reduction of Regnase-1 expression in HSPCs leads to the development of pathological hematopoiesis.

Loss of Regnase-1 leads to aberrant differentiation. We next tested whether loss of Regnase-1 in HSPCs relates to the development of leukemia. Aberrant blast cells with nucleoli were observed in the PB of 8 -week-old Reg $1^{\Delta / \Delta}$ mice and were also seen in the BM (Fig. 5a, b, Supplementary Fig. 4a, b). Mononuclear cells in PB and BM were analyzed further to reveal the variations in disease phenotype. In $\mathrm{PB}$ from $\operatorname{Reg} 1^{\Delta / \Delta}$ mice, we observed $1-14 \%$ of lymphoblasts, $16-22 \%$ of atypical lymphocytes exhibiting abnormal nuclear morphology, 2-4\% of mature myelocytes and metamyelocytes, and $1-16 \%$ of atypical cells including stab cells in the neutrophil population. In addition, $3-8 \%$ of monoblasts and $6-20 \%$ of atypical mononuclear cells were seen, and proerythroblastic-polychromatophilic erythroblasts were also observed. Furthermore, $1 \%$ of myeloblasts was present, and total white blood cell counts were increased; therefore we diagnosed abnormal hematopoiesis, such as MPN (Fig. 5c, d). Reg1 ${ }^{\Delta /+}$ mice also had a similar phenotype (Supplementary Fig. 4c). BM of $\operatorname{Reg}^{\Delta / \Delta}$ mice manifested no significant differences in frequencies of neutrophil populations, but had an expanded monocyte and reduced lymphocyte population. In addition, morphologically abnormal cell populations with increased cell volume or irregular nuclear shape were present in the leukocyte population i.e., neutrophils, monocytes, and lymphocytes, in Reg1 ${ }^{\Delta / \Delta}$ mouse BM (Supplementary Fig. 4d). Consistent with abnormalities in the mononuclear cells, LSK HSPCs from $\operatorname{Reg} 1^{\Delta / \Delta}$ mice displayed enlarged nuclei and aberrant cell shapes (Fig. 5e). These results suggest that Regnase- 1 is required to maintain normal hematopoiesis and that disrupted expression of Regnase-1 leads to aberrant differentiation. In recipients transplanted with MNCs from BM of $\operatorname{Reg} 1^{\Delta / \Delta}$ mice, abnormal proliferation of donor-derived HSPCs, splenomegaly, and an abnormal hematopoietic phenotype of $\mathrm{PB}$ was observed, but blasts were not present in the $\mathrm{PB}$. These results indicate that Reg1 ${ }^{\Delta / \Delta}$ mouse-derived aberrant cells are transplantable (Supplementary Fig. 5a-d). The phenotype of Regnase-1-deficient 
a

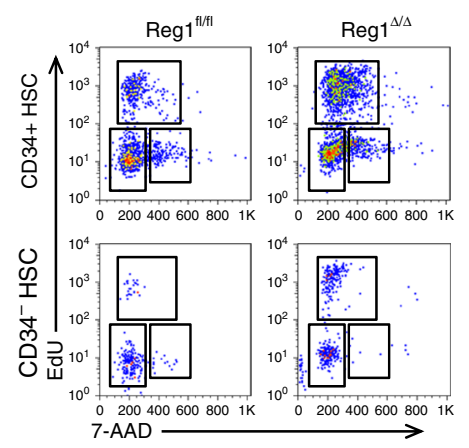

e

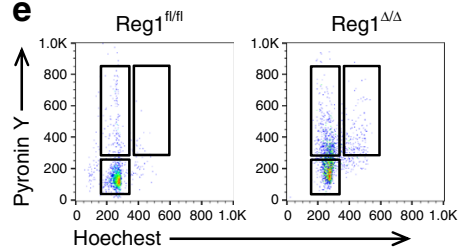

g

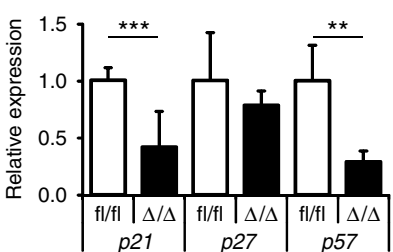

b
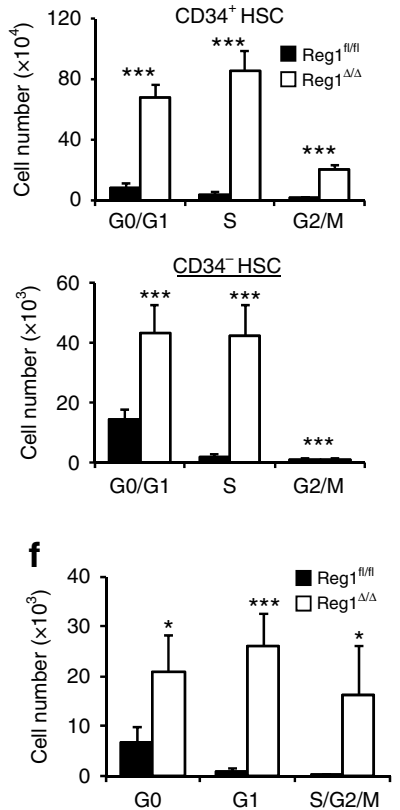

h

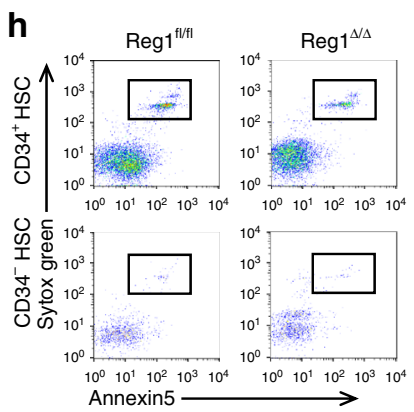

$\mathbf{k}$

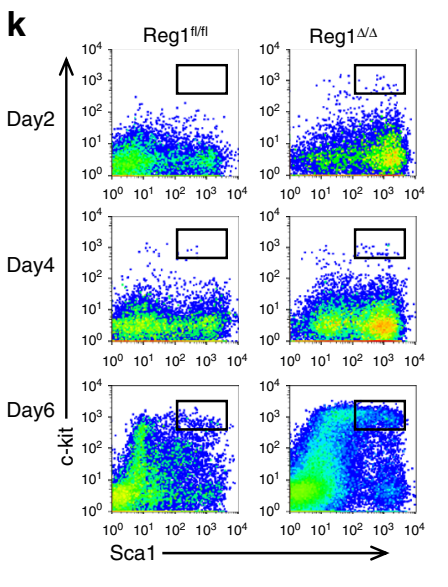

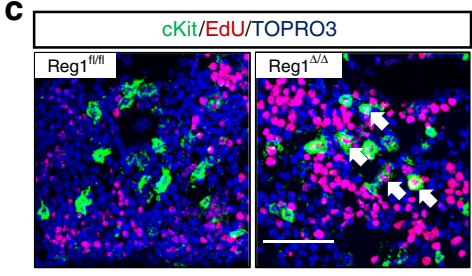
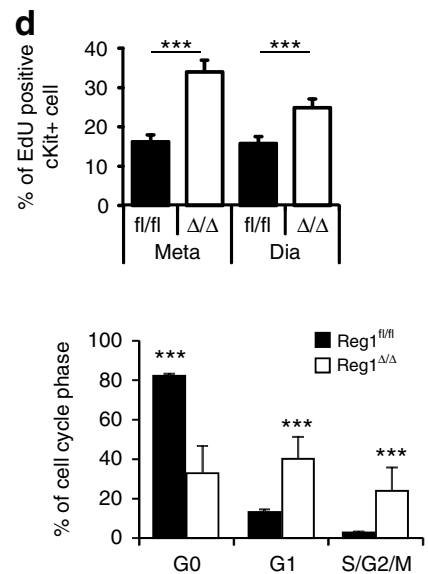

i

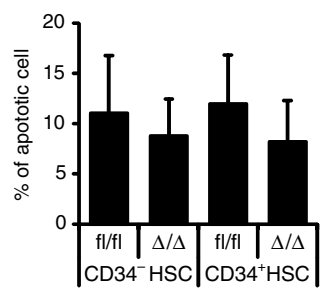

I

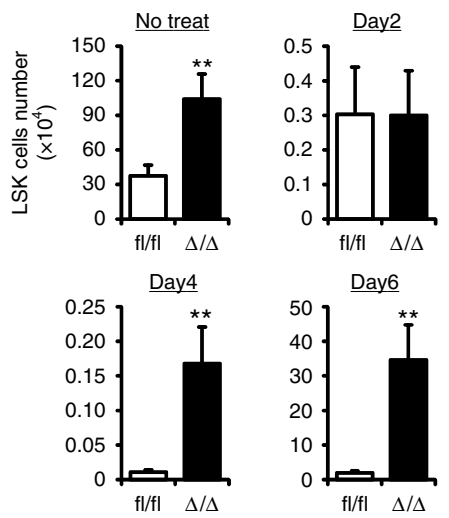

mice is not completely consistent with AML pathology, but does exhibit aberrant differentiation and proliferation of the HC.

To confirm the relevance of Regnase-1 deficiency for AML pathogenesis, we undertook RNA-seq-based genome-wide gene expression profiling using CD34- HSCs collected from control Reg $1^{\text {flox/flox }}$ and Reg $1^{\Delta / \Delta}$ mice BM. RNA-seq revealed that the expression of many genes was altered in Reg1 ${ }^{\Delta / \Delta}$ mice (Fig. 5f). Consistent with the morphological analysis, gene set enrichment analysis (GSEA) revealed that the genes downregulated in CD34HSC following Regnase-1 deletion were statistically significantly similar to an a priori-defined set of genes in HSCs from Pu.1-deficient mice, taken to model AML (Fig. 5g). A comparison of gene expression sets between CD34- HSCs from Regnase-1deleted mice and BM from human AML-M1, M2, and M3 patients indicated significantly similar concordant differences in gene expression between Regnase-1-deleted CD34- ${ }^{-}$HSCs and human AML cells (Fig. 5h). Although Regnase-1 mRNA expression was not associated with prognosis, patients whose AML cells exhibited gene expression profiles similar to Regnase1-deficient $\mathrm{CD}^{-} 4^{-}$HSCs had a poor prognosis (Fig. 5i, 
Fig. 3 Loss of Regnase-1 expression results in accelerated CD34- HSC cell cycle progression. a Cell-cycle analysis of control (fl/fl) or Regnase-1-KO ( $\Delta / \Delta)$ BM CD34- HSC and CD34+ HSC populations using EdU/7AAD staining. Dot plots indicating the frequency of HSPCs in G0/G1 (EdU- 7AAD ${ }^{-}$), G2/M $\left(\mathrm{EdU}^{+}\right)$, and $\mathrm{S}\left(\mathrm{EdU}+7 \mathrm{AAD}{ }^{+}\right)$phase of cell cycle $(n=3$ per group; 3 independent experiments). $\mathbf{b}$ Quantification of HSPCs as shown in $\mathbf{a}$. $\mathbf{c}$ Detection of proliferative cells in BM section of control $(\mathrm{fl} / \mathrm{fl})$ or Regnase-1-KO $(\Delta / \Delta)$ mice by EdU labeling. Representative image showed EdU staining together with cKit and Nuclei (TOPRO3) counter-staining. Scale bars represent $50 \mu \mathrm{m}$. d Quantification of the ratio of EdU-positive proliferating cells in c-Kit-positive cells. e Representative cell cycle plots of CD34- HSC populations in BM from control (fl/fl) or Regnase-1-KO $(\Delta / \Delta)$ mice using Hoechst 33342/pyronin $\mathrm{Y}$ staining ( $n=3$ per group; 3 independent experiments). $\mathbf{f}$ Representative data showing number and ratio of cells in the G0, G1, and S/G2/M phases as shown in e. $\mathbf{g}$ qRT-PCR analysis of cell cycle regulatory genes in CD34- HSCs from control $(\mathrm{fl} / \mathrm{fl})$ and Regnase-1-KO $(\Delta / \Delta)$ mice $(\mathrm{n}=3$ per group). $\mathbf{h}$ Flow cytometric analysis of apoptotic populations in CD34- $\mathrm{HSCs}$ and CD34+ $\mathrm{HSCs}$ of control $(\mathrm{fl} / \mathrm{fl})$ or Regnase-1-KO $(\Delta / \Delta)$ mice BM with a combination of annexin $\mathrm{V}$ and SYTO Green staining ( $n=3$ per group; 3 independent experiments). i Ratio of apoptotic cells (annexin $\mathrm{V}^{+}$SYTO Green ${ }^{+}$). Data are the means \pm SD. j Kaplan-Meier survival curve of control (fl/fl) and Regnase-1-KO $(\Delta / \Delta)$ mice after weekly administration of $5-\mathrm{FU}(150 \mathrm{mg} / \mathrm{kg})(n=7)$. $P$ values were calculated by the logrank test. $\mathbf{k}$ Representative dot plot depicting frequency of phenotypically defined HSPCs at the indicated days following a single injection of 5-FU. I Absolute LSK cell numbers from control $(\mathrm{fl} / \mathrm{fl})$ and Regnase-1-KO $(\Delta / \Delta)$ mice at the indicated days after 5-FU treatment $(n=3)$. $\mathbf{m}$ Expression Regnase-1 mRNA in LSK cells of control $(\mathrm{fl} / \mathrm{fl})$ mice at the indicated days after 5 -FU treatment $(n=3)$. Error bars indicate mean \pm SD. ${ }^{\star} p<$ $0.05 ;{ }^{\star \star} p<0.01,{ }^{\star \star \star} p<0.005$, two-sided $t$-test in $\mathbf{b}, \mathbf{d}, \mathbf{f}, \mathbf{g}, \mathbf{i}, \mathbf{m}, \mathbf{I}$

Supplementary Data 1). In addition, we found that overexpression of Regnase- 1 suppresses the exuberant growth of THP1 and HL60 cells (Fig. 5j).

Regnase-1 regulates the stability of Gata2 and Tal1 mRNA. Regnase- 1 has been reported to control target gene expression by recognizing the specific $3^{\prime} \mathrm{UTR}$ elements of mRNA and to facilitate ribonuclease-mediated degradation ${ }^{31}$. To identify Regnase- 1 target mRNAs in CD34- HSCs, we constructed luciferase reporter vectors by inserting the $3^{\prime}$ UTRs of candidate mRNAs into the $3^{\prime}$ site of the luciferase expression cassette and then determining whether these $3^{\prime}$ UTRs were actually Regnase- 1 binding sites for degradation. Because Regnase-1 degrades various target mRNAs ${ }^{32}$ and RNA-Seq analysis showed that the expression of numerous genes is affected by Regnase-1 deficiency (Fig. 5f), we focused on transcription factors that affect the expression of a wide range of genes important for hematopoiesis, such as Gata2, Tal1, Bmi1, Ctnnb1, Runx1, Hoxb4, and Egr1. In accordance with a previous report ${ }^{33}$, co-transfection of luciferase reporter vectors of candidate genes together with the Regnase- 1 expression vector resulted in suppression of luciferase activity caused by the RNase-activity of Regnase-1, relative to cotransfection with Regnase-1 lacking the RNase activity vector (Fig. 6a, Supplementary Fig. 6). Next, the identity of the candidate genes affected by Regnase-1 overexpression was confirmed by real-time QPCR. We concluded that Gata2 and Tal1 were prime targets of Regnase-1, because expression of these mRNAs was significantly upregulated in CD $34^{-}$HSCs from Reg1 ${ }^{\Delta / \Delta}$ mice (Fig. 6b). To confirm that the degradation of Gata2 and Tal1 mRNA is directly induced by Regnase-1, we performed Northern blotting. Overexpressing Regnase-1 in THP1 cells attenuated Gata2 and Tal1 mRNA expression by accelerating the degradation of these mRNAs, but did not alter CD34 mRNA degradation. (Fig. 6c, Supplementary Fig. 7a). Moreover, the half-lives of Gata2 and Tal1 mRNA were extended in Regnase-1-deficient CD34HSCs relative to CD34- HSCs from control mice (Fig. 6d). In summary, our findings suggest that Gata2 and Tal1 identified by bioinformatics analysis are indeed recognized and degraded by Regnase-1.

Regnase-1 regulates degradation of Gata2 and Tal1 mRNA. Next, we investigated whether inhibition of Gata2 and Tal1 in CD34- HSCs of Reg1 ${ }^{\Delta / \Delta}$ mice rescues the excessive HSPC selfrenewal and subsequent development of aberrant differentiation. We did this by infection with an shRNA virus and confirmed knock-down of the expression of the targeted gene (Fig. 7a). To investigate the effect of Gata2 and Tall knock-down on the proliferation and differentiation of $\operatorname{Reg} 1^{\Delta / \Delta}$ CD34- HSCs, we performed colony-forming assays. As expected, shRNA-mediated suppression of either or both Gata2 and Tal1 expression partially rescued the colony-forming ability of CD34- HSCs from $\operatorname{Reg} 1^{\Delta / \Delta}$ mice, whereas scrambled control shRNA had no effect (Fig. 7b). However, knock-down of Bmil (non-candidate control gene) did not alter the colony forming ability of Reg1 $1^{\Delta / \Delta}$ CD $34^{-}$HSCs, suggesting that aberrant proliferation of these CD34- HSCs is induced by excessive expression of Gata2 and Tall. In addition, we investigated the effect of shRNAinduced knockdown of Gata2 and Tal1 for leukemia development in vivo. Upon transplantation, engrafted Gata2 and Tal1 knockdown LSK cells from Reg1 ${ }^{\Delta / \Delta}$ BM showed moderate LSK-HSC or CD34- HSC expansion compared to controls (Fig. 7c-f). Correspondingly, Gata2 and Tal1 down-regulation partially alleviated splenomegaly and mesenteric lymphadenopathy (Fig. $7 \mathrm{~g}$ ). We investigated whether Gata2 and Tal1 overexpression is involved in the observed cell cycle abnormalities of Regnase-1-deficient HSPCs. Cell cycle abnormalities observed in CD34- HSCs due to Regnase-1 deficiency were partially recovered by knock-down of Gata2 and Tal1 expression (Fig. 7h). In summary, our in vitro and in vivo data indicate that dysfunction of Regnase-1 causes hyperactive Gata2 and Tal1 function, resulting in exuberant self-renewal of HSPCs and aberrant differentiation.

\section{Discussion}

HSPC homeostasis must be maintained by proper regulation of intracellular signaling for the continuous provision of blood cells throughout life. The cell fate of HSPCs, self-renewal, quiescence, and differentiation is decided by a network of transcription factors $^{34,35}$. However, the regulatory mechanisms responsible for the intricate coordination of transcription factors in HSPCs according to the situation has not been investigated. Here, we identified Regnase-1 as a molecule expressed in HSPCs and inversely correlated with their proliferative capacity. A previous report had shown that Regnase-1 modulates immune responses by degrading inflammatory cytokine mRNAs in macrophages and T lymphocytes ${ }^{14}$. Using mice lacking Regnase-1 in hematopoietic cells, we report that this factor controls proliferation and differentiation of CD34- HSCs and is also involved in homeostasis of myelopoiesis. A significant expansion of Regnase-1-deficient CD34- HSCs was provoked through promoting cell cycle progression, accompanied by an aberrant expression of cyclindependent kinase inhibitory proteins. Importantly, Regnase-1deficient mice exhibited abnormal hematopoiesis including a marked accumulation of abnormal blasts in the blood, lethal within three months of age. Mechanistically, we found that 
a

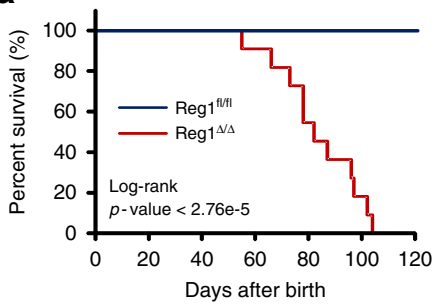

b

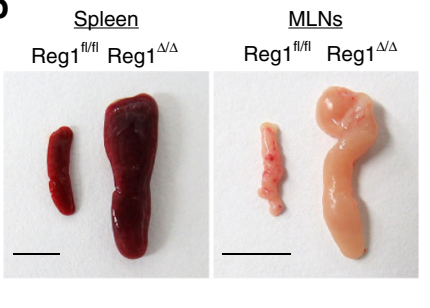

C

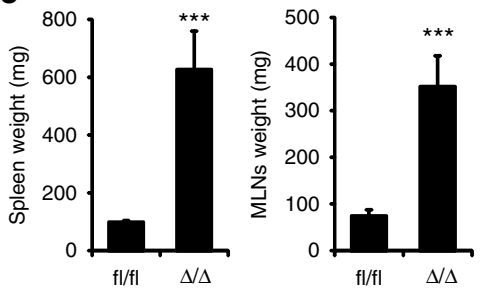

d

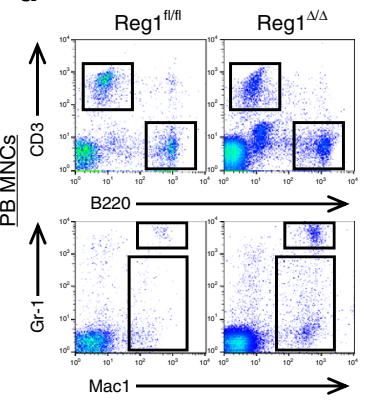

e

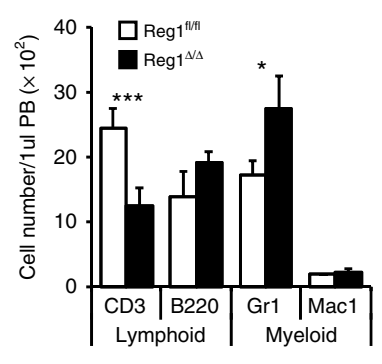

f

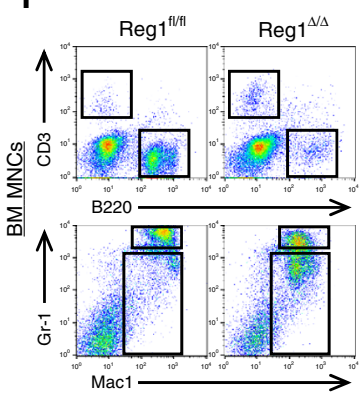

g

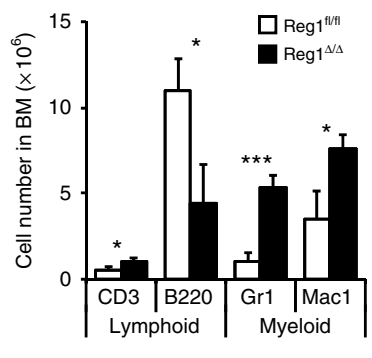

h

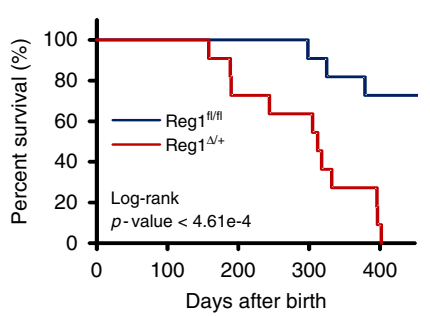

k

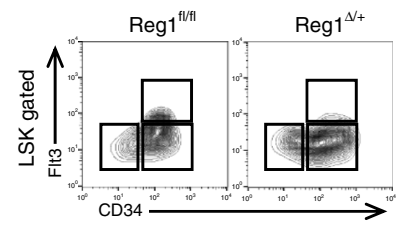

I

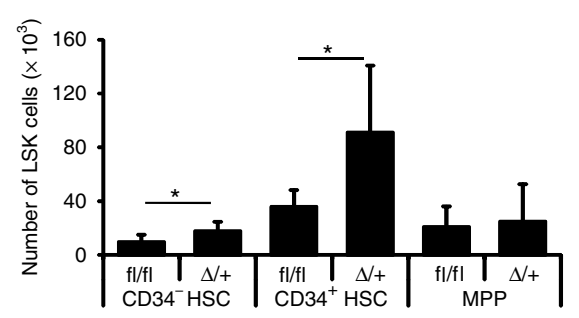

i

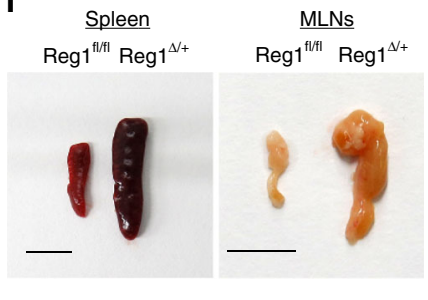

j

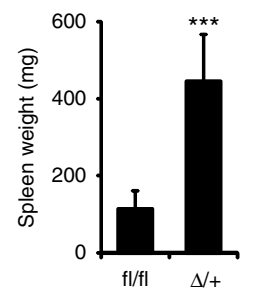

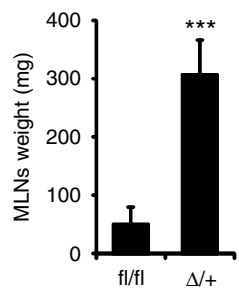

$\mathbf{m}$
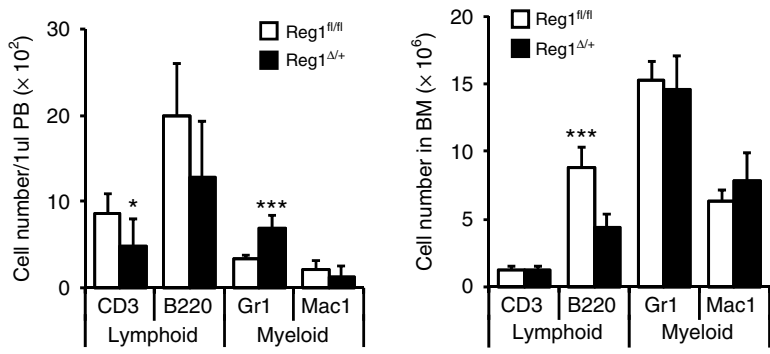

Fig. 4 Loss of Regnase1 causes impaired hematopoietic functions. a Kaplan-Meier survival curve of control $(\mathrm{fl} / \mathrm{fl})$ and Regnase-1-KO $(\Delta / \Delta)$ mice $(n=11)$. $P$ values were calculated by the logrank test. $\mathbf{b}$ Representative gross appearance of the spleen and mesenteric lymph nodes (MLNs) collected from 8-weekold mice as indicated. The scale bars represent $1 \mathrm{~cm}$. c Spleen (right) and MLNs (left) weight of 8 -week-old mice as indicated ( $n=4$ per group).

d Flow cytometric analysis of lymphocytes and monocytes in PBMCs from 8-week-old mice as indicated ( $n=3$ per group; 3 independent experiments). e Quantification of frequencies of lymphoid and myeloid cells in PBMCs. f Flow cytometric analysis of lymphocytes and monocytes in BM MNCs from 8week-old mice as indicated ( $n=3$ per group; 3 independent experiments). $\mathbf{g}$ Quantification of frequencies of lymphoid and myeloid cells in bone marrow

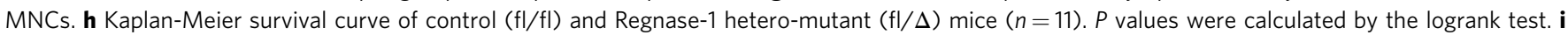
Representative images of the spleen and MLNs collected from 12-month-old mice as indicated. The scale bars represent $1 \mathrm{~cm}$. $\mathbf{j}$ Spleen (right) and MLNs (left) weight of 12-month-old mice as indicated ( $n=4$ per group). k, I Representative flow cytometric analysis and quantitative evaluation of LSK-HSC numbers in the BM of 8 -week-old mice as indicated ( $n=3$ per group; 3 independent experiments). $\mathbf{m}$ Quantification of ratios of lymphocytes and

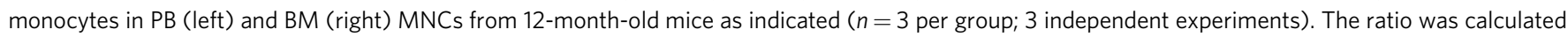
by flow cytometric analysis. Error bars indicate mean \pm SD. ${ }^{\star} p<0.05 ;{ }^{\star \star} p<0.01,{ }^{\star \star \star} p<0.005$, logrank test in $\mathbf{h}$, two-sided $t$-test in $\mathbf{c}, \mathbf{e}, \mathbf{g}, \mathbf{j}, \mathbf{m}, \mathbf{I}$

Regnase-1 targets the 3'UTR of mRNAs encoding Gata2 and Tall, and post-transcriptionally modulates their activity through mRNA degradation. Excessive proliferation of HSPC resulting from Regnase-1 deficiency may allow progression to abnormal hematopoiesis by promoting mutagenesis. Collectively, our data reveal a role for Regnase-1 in regulating HSPC proliferation in hematopoietic homeostasis, the dysfunction of which could lead to the generation of leukemic cells. 
a

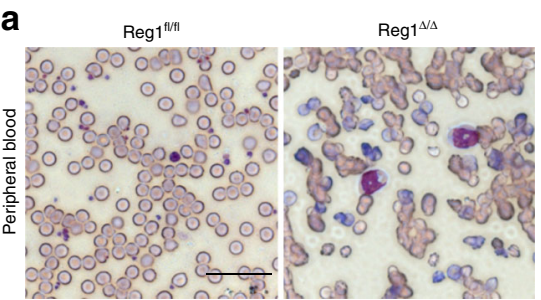

b

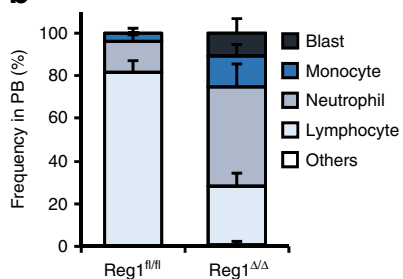

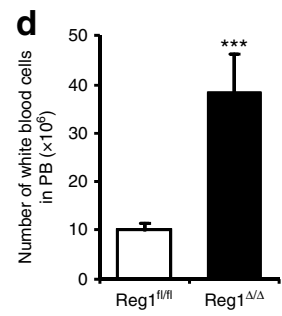

C

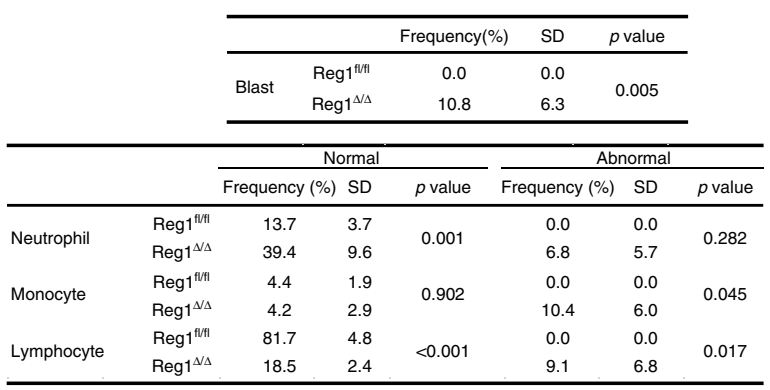

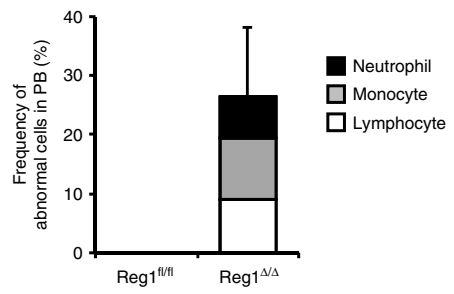

e
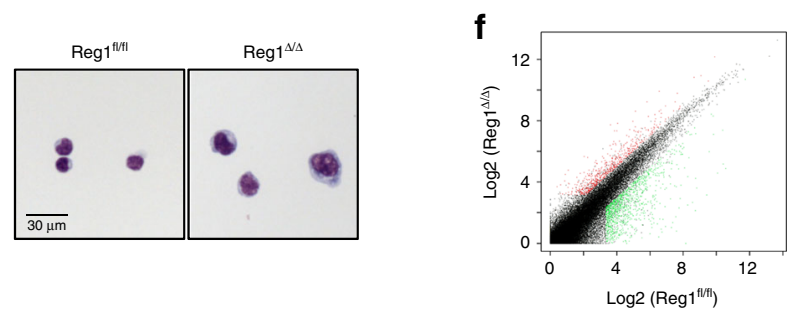

g moue HSC of Pu.1 deletion Enrichment plot: GSEE654.PU.1.1KO__HSC_VS.PU.

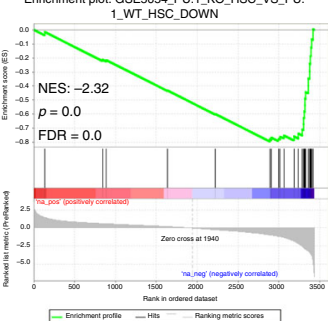

h
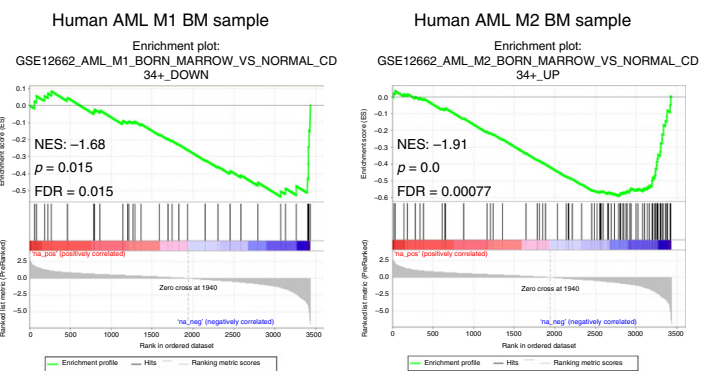

Human AML M3 BM sample GSE12662_AML_M3_BORN_MARROW_VS_NORMAL_CD

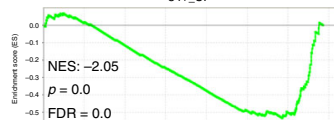

i

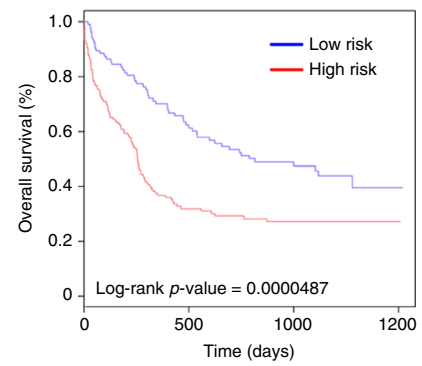

j
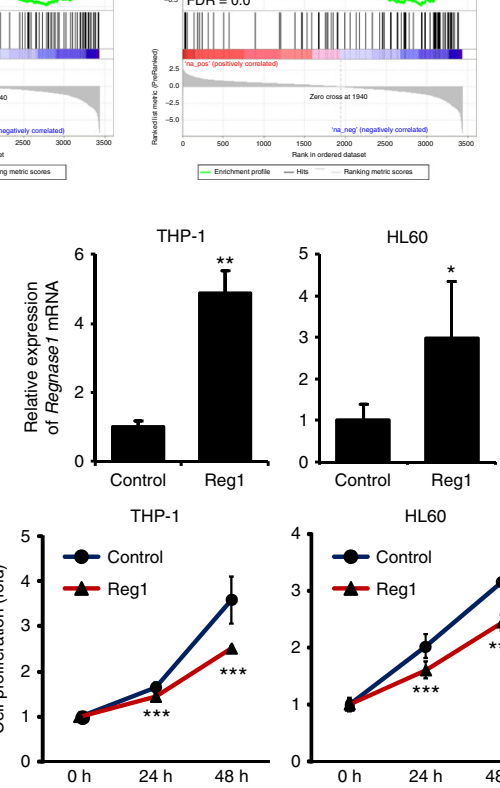

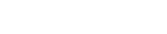

The fate of hematopoietic cells is controlled through transcription factor-mediated gene expression. Thus far, many transcription factors related to HSPC development have been identified, and their functional roles clarified by studies using genetically modified mice ${ }^{36,37}$. However, hematopoiesis in the living organism is not controlled by the simple presence or absence of transcription factors, but by intricately refined tuning of their activities. In addition, gene regulatory networks involving interactions of many different combinations of transcription factors with diverse activities ensure robustness and adaptability of HSPC function. In our study, we found that Regnase-1 degrades Gata2 and Tal1 mRNA, which encode transcription 
Fig. 5 Regnase-1-deleted HSPCs show a phenotype and gene expression profile similar to leukemia. a Representative field of Wright-Giemsa-stained BM smears of 8-week-old control (fl/fl) and Regnase-1-KO $(\Delta / \Delta)$ mice. The scale bar shows $30 \mu \mathrm{m}$. $\mathbf{b}$ Quantification of frequencies of blasts and other MNCs in PB on Wright-Giemsa-staining ( $n=3$ per group; 3 independent experiments). c Quantification of abnormal cells in the PB. $\mathbf{d}$ Peripheral white blood cell counts in blood samples from 8-week-old control (fl/fl) or Regnase-1-KO $(\Delta / \Delta)$ mice $(n=3$ per group). e Wright-Giemsa staining of CD34- HSCs. The scale bars represent $30 \mu \mathrm{m}$. f Scatter-plot representation of the transcriptional differences between control Reg flox/flox $(\mathrm{fl} / \mathrm{fl})$ and $\mathrm{Vav1-iCre;}$ Regnase- $\mathrm{f}^{\text {flox } / \text { flox }}(\Delta / \Delta)$ CD34- HSCs. The color indicates $>2$-fold differential gene expression $(n=3$ per group and $n=2$ per group; 2 independent experiments; the average of the two is shown). $\mathbf{g}$ Gene set enrichment analysis comparing CD34- HSCs from control ( $\mathrm{fl} / \mathrm{fl})$ and Regnase-1-KO $(\Delta / \Delta)$ mice with the association between genes upregulated following Regnase-1 deletion and Pu.1 deletion. $\mathbf{h}$ Human HSC signatures of AML compared with Regnase1-deficient CD34- HSCs. The normalized enrichment scores (NES), P-value and $q$-value (FDR) are indicated on each plot. i Kaplan-Meier plot of AML patient survival data based on similarity of gene expression profiles of loss of Regnase-1. $P$ values were calculated by the logrank test. $\mathbf{j}$ Regnase- 1 mRNA expression (upper panel) and proliferation (lower panel) of THP1 and HL60 leukemic cells transfected with mouse Regnase-1 cDNA expression vector ( $n=$ 3 per group; 3 independent experiments). Error bars indicate mean \pm SD. ${ }^{\star} p<0.05 ;{ }^{\star \star} p<0.01,{ }^{\star \star \star} p<0.005$, log rank test in $\mathbf{i}$, two-sided $t$-test in $\mathbf{c}$, $\mathbf{d}, \mathbf{j}$

factors essential for HSPC integrity. Loss of function of Regnase-1 in HSPCs results in up-regulation of Gata2 and Tal1 expression and leads to excessive self-renewal. These findings suggest that Regnase- 1 could have a role in fine tuning the transcription factor network by post-transcriptional regulation. Regnase-1 is a member of the $\mathrm{CCCH}$ zinc finger protein family harboring a PIN domain with RNAse activity. Several studies have demonstrated that Regnase- 1 acts as a transcription factor regulating apoptosisrelated gene expression and that it has a role in miRNA biogenesis by cleaving target pre-miRNAs ${ }^{38,39}$. Recently, it was reported that Regnase-1 has post-transcriptional regulatory activity through recognition of the specific $3^{\prime} \mathrm{UTR}$ sequence of the target mRNA and induction of mRNA degradation via its RNAse activity, but data on the binding sequence of Regnase- 1 and mechanism of RNA cleavage are still limited ${ }^{40,41}$. In macrophages, Regnase- 1 controls the immune response by degrading mRNAs encoding $I L-1 \beta, I L-6$, and $I L-12 p 40$ mRNA $^{16}$, whereas in $\mathrm{T}$ cells, it targets $\mathrm{mRNAs}$ encoding immunoregulatory proteins such as $c$-Rel, OX40, and $I L-2^{16}$. Because the target mRNA varies depending on cell type, we showed that Gata2 and Tal1 degradation is specific for CD34- HSCs, although inflammatory cytokine expression was not significantly altered in Regnase-1-deleted HSPCs. Regnase-1 deficiency in immune cells such as macrophages and T-cells causes a systemic inflammatory and severe autoimmune disease phenotype ${ }^{14,16}$. Our results show that Regnase-1 deletion in HSPCs leads to their excessive proliferation and aberrant differentiation. Therefore, Regnase- 1 regulates specific mRNA expression in each blood cell type and functions in a context-dependent manner. On the other hand, because Cremediated Regnase- 1 deletion occurred not only in HSPCs but also lineage-committed cells in Vav1-iCre; Regnase- $1^{\text {flox/flox }}$ mice, phenotypes in mutant mice are not exclusively caused by abnormal HSPCs. The cell type-specific regulatory mechanism of Regnase-1 might involve cell-specific functions of this factor. During an inflammatory response, Regnase-1 expression is regulated by Toll-like receptor-mediated and pro-inflammatory signals in macrophages or T-cell receptor-mediated signals in $\mathrm{T}$ cells $^{16}$. For HSPC maintenance, the regulatory mechanism of expression and function of Regnase-1 has remained unclear. Further precise analysis may unmask regulatory mechanisms responsible for Regnase- 1 activity by analyzing the signals from the BM microenvironmental niche.

The transcription factors Gata2 and Tal1/SCL are expressed in HSPCs and are implicated in hematopoietic differentiation and proliferation $^{42,43}$. Either reduction or gain of Gata2 function caused by genetic alteration leads to abnormal hematopoiesis and predisposes to AML. Lower amounts of Gata2 caused by mutations contribute to the development of myelodysplastic syndrome (MDS) and $\mathrm{AML}^{44}$. Gata2 is highly expressed in AML and its overexpression through $\mathrm{p} 38$ /ERK-dependent signaling portends a poor prognosis of cytogenetically normal AML ${ }^{45,46}$. Gene knockdown or pharmacological inhibition of Gata2 in AML cells can relieve drug resistance by inducing an increase of the apoptotic population. (Yang, L., Sun, H., Cao, Y. PLoS One (2017)). Tall is also known to act as a proto-oncogene, and aberrant Tall transcription is related to the pathogenesis of $\mathrm{T}$ cell acute lymphoblastic leukemia (T-ALL) ${ }^{47,48}$. Aberrant expression of Tal1 in T-ALL represses the expression of genes controlling cell homeostasis $^{49,50}$. We found that deletion of Regnase-1 in HSPCs causes aberrant differentiation by inducing overexpression of molecules that maintain normal hematopoiesis, such as Gata2 and Tal1. In our experiments, however, knockdown of Gata2 and Tal1 in Regnase-1-deleted HSPCs was not sufficient to fully normalize hematopoiesis. Therefore, other Regnase-1 target mRNAs are likely to exist. In addition, no direct binding of Gata2 or Tal1 to p21 or p57 promoter regions was found using chromatin immunoprecipitation assays (Supplementary Fig. 7b); thus, further exploration of the regulatory mechanisms of p21 and p57 expression by Gata2 and Tal1 is required. Further work is required to unveil the mechanism of leukemogenesis.

Regnase-1 acts as a fine-tuning regulator of global transcription factors not only for physiological hematopoiesis but also for leukemogenesis. Emerging evidence from this research using Regnase-1 knock-out mice suggests that this molecule has potential relevance for human AML. Bioinformatics analysis revealed that gene expression sets of Regnase-1-deleted mouse HSPCs were significantly enriched in the group of human AMLs tested, but gene mutations and mRNA alterations of Regnase-1 in human AML patients had not been reported thus far. Therefore, Regnase-1 expression may be regulated by protein modification such as phosphorylation and ubiquitination. Regnase-1 is a powerful tumor suppressor gene, decreased amounts of the product of which results in the spontaneous development of abnormal hematopoiesis even in heterozygous mice. This may provide an opportunity for drug discovery for the treatment of AML. Previous studies in $\mathrm{CD}^{+}{ }^{+} \mathrm{T}$ cells showed that Regnase- 1 expression and function is suppressed by MALT1, which possesses an arginine-specific protease activity ${ }^{16,51}$. MALT1 inhibitors are therefore possible candidates for enhancing Regnase-1 expression, suggesting their potential as therapeutic agents in inflammatory diseases and viral infections ${ }^{52,53}$. For therapeutic applications, it will be necessary to elucidate the molecular mechanisms which could be employed to enhance or repress Regnase-1 activity in leukemic cells.

In summary, here we have shown that Regnase- 1 contributes to hematopoietic homeostasis by post-transcriptional regulation of target mRNA stability. Regnase-1 may simultaneously modulate the expression of numerous essential factors for HSPC selfrenewal and differentiation including Gata 2 and Tal1. We suggest that Regnase-1, a post-transcriptional regulator, is a causative molecule for the development of abnormal hematopoiesis, and that understanding of the regulatory mechanisms controlling 

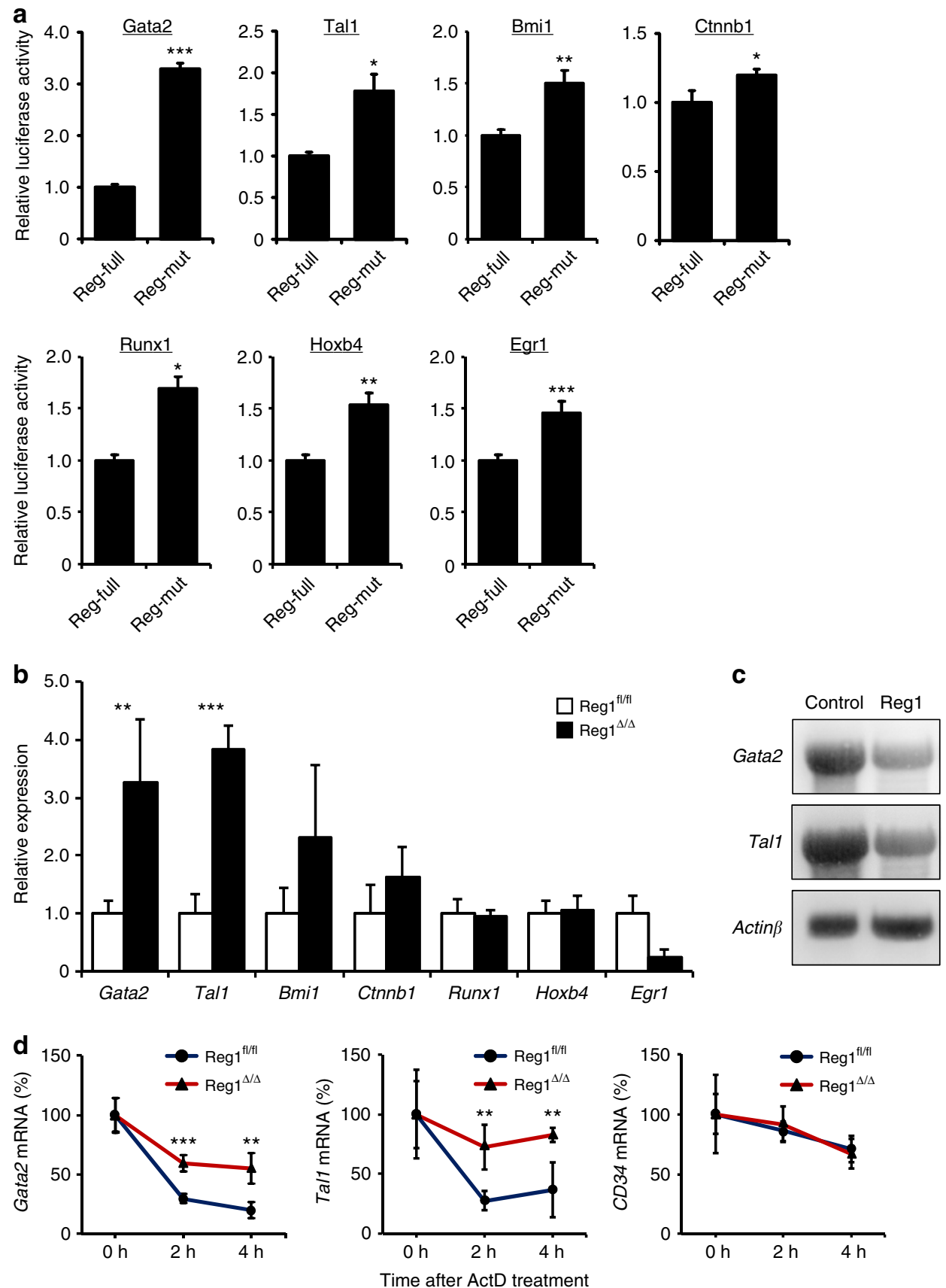

Fig. 6 Regnase-1 recognizes and degrades Gata2 and Tal1 mRNA. a Luciferase activity of HEK293 cells transfected with luciferase reporter plasmids containing $3^{\prime}$ UTRs of the indicated genes and either Regnase-1 expression plasmid or inactive Regnase-1 mutant plasmid (3 independent experiments). b qRT-PCR analysis of candidate gene expression in CD34- HSCs from control (fl/fl) or Regnase-1-KO $(\Delta / \Delta)$ mice BM ( $n=3$ per group; 3 independent experiments). c THP1 cells were transfected with human Regnase-1 cDNA expression vector or control (empty) vector. After 48 h, levels of Gata2, Tal1, and Actin $\beta$ mRNA were determined by Northern blotting. Representative data of two independent experiments are shown. d Quantitative RT-PCR of Gata2, Tal7, and CD34 expression in CD34- HSCs from control (fl/fl) or Regnase-1-KO $(\Delta / \Delta)$ mouse BM treated for 0-4 h with actinomycin D (ActD) ( $n=3$ mice per group). Data are expressed as fold-change relative to $0 \mathrm{~h}$ treatment. Error bars indicate mean $\pm \mathrm{SD} .{ }^{\star} p<0.05$; ${ }^{\star \star} p<0.01,{ }^{\star \star \star} p<0.005, \mathrm{two}-\mathrm{sided} t$-test in $\mathbf{a}, \mathbf{b}, \mathbf{d}$

Regnase- 1 expression and identifying additional target genes will provide valuable information for therapies targeting AML.

\section{Methods}

Mice. C57BL/6 mice (B6-Ly5.2) were purchased from Japan SLC (Shizuoka, Japan) and C57BL/6-Ly5.1 mice were purchased from Sankyo Labo Service (Tsukuba, Japan) and used between 6 and 12 weeks of age. Regnase-1 floxed mice were generated as previously described ${ }^{16}$. A2Kio/J (Vav1-iCre) mice were purchased from The Jackson Laboratory (Bar Harbor, ME). Animals were housed in environmentally controlled rooms of the animal experimentation facility at Osaka University. All experiments were carried out under the guidelines of Osaka University Committee for animal and recombinant DNA experiments and were approved by the Osaka University Institutional Review Board.

Cell culture and transfections. Human leukemia cell lines (HL60 and THP-1) and HEK293 cells were provided by the RIKEN cell bank. Cells were maintained in RPMI-1640 medium (SIGMA) or DMEM medium (SIGMA) supplemented with $10 \%$ heat-inactivated fetal bovine serum (SIGMA). Transfections of Regnase-1 
a

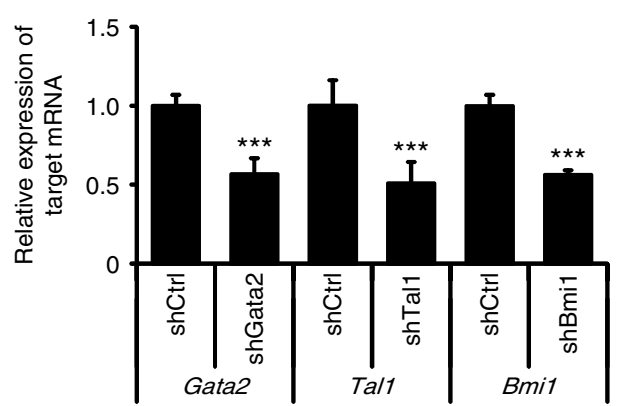

b
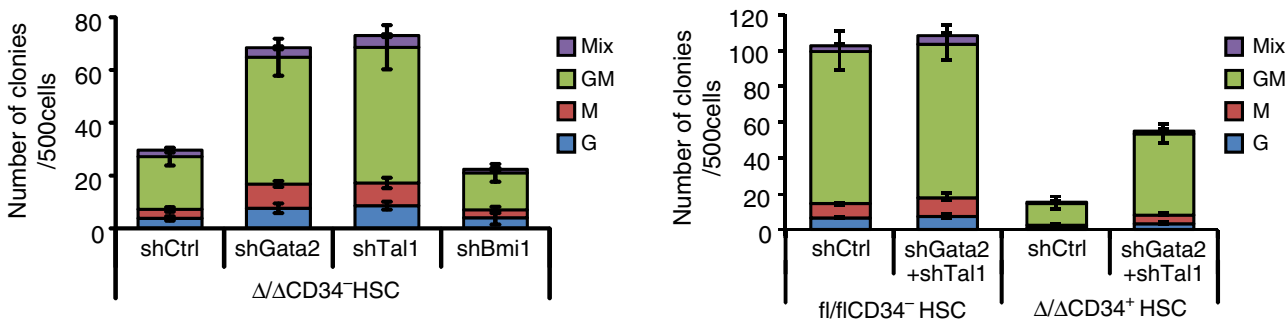

C
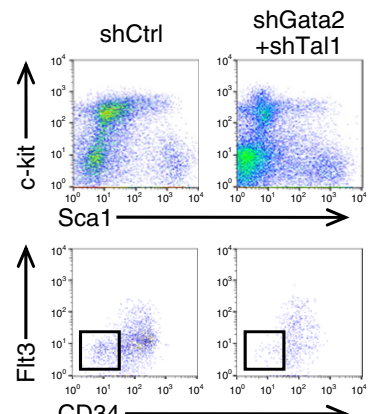

d

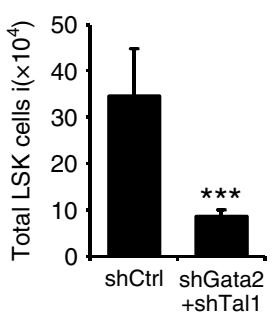

e

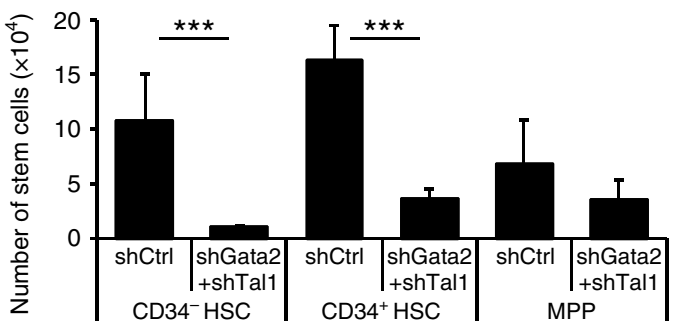

f

g
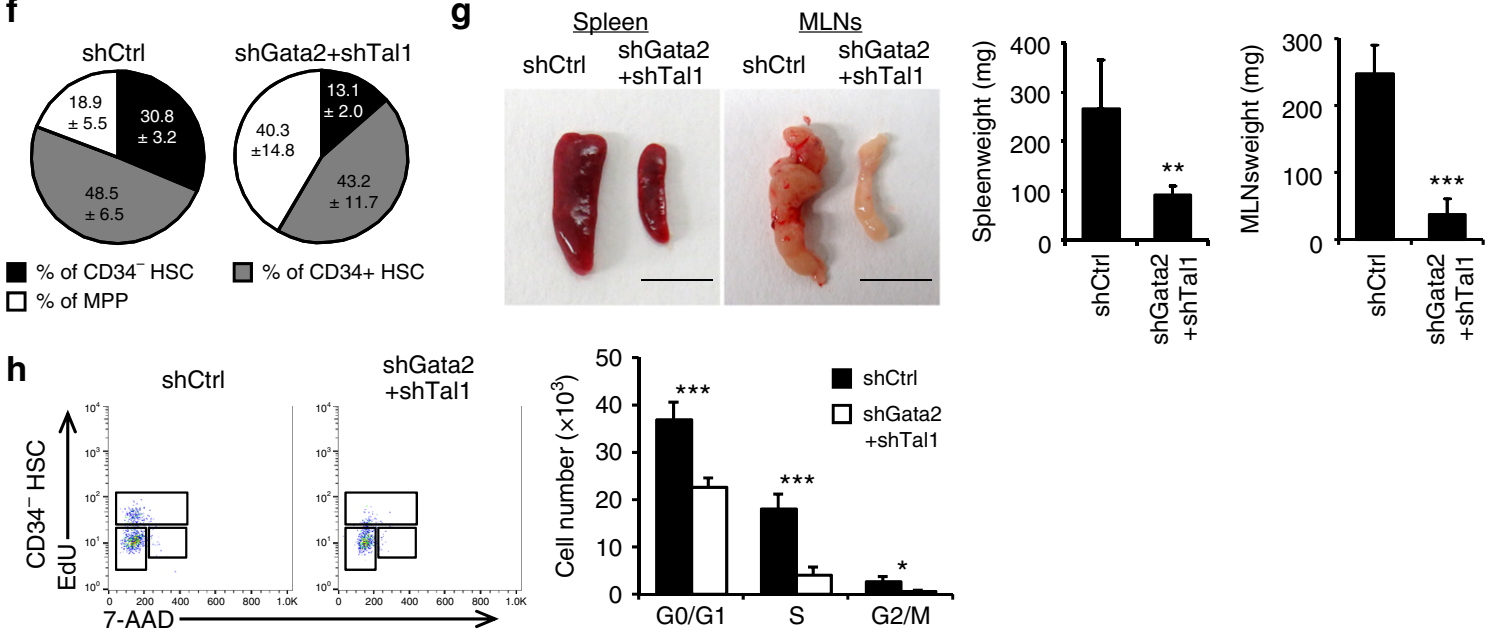

expression vector ${ }^{33}$ or empty vector (pcDNA3) were performed using FuGENE HD transfection reagent (Promega) according to manufacturer's instructions. Proliferation of HL60 and THP-1 cells was evaluated using Cell Counting Kit-8 (Dojindo). All of the cells were authenticated by examination of morphology and growth characteristics, and were confirmed to be mycoplasma-free.

Immunohistochemical analysis. Tissue fixation and staining of sections or tissues with antibodies was performed by the following method. Freshly-excised femoral or tibial bone from WT mice was embedded in super cryoembedding medium (Leica Microsystems) and frozen in liquid nitrogen. Next, $10-\mu \mathrm{m}$ cryosections were generated via the Kawamoto film method ${ }^{54}$. An anti-c-Kit (AF1356, R\&D Systems, dilution 1/100), anti-Scal (553333, BD Biosciences, dilution 1/100), anti-CD71 (113802, Biolegend, dilution 1/100) and, anti-Regnase-1 antibodies (dilution $1 / 100)^{16}$ were used for staining ${ }^{55}$. Cell nuclei were visualized with TO-PRO-3 and sections were examined by confocal microscopy (TCS/SP8; Leica). EdU labeling and staining was performed using a Click-iT ${ }^{\circledast}$ Plus EdU Alexa Fluor 555 Imaging Kit (Thermo Fisher) according to the manufacturer's instructions. In all assays, isotype-matched Ig was used as a negative control and it was confirmed that the positive signals were not derived from nonspecific background. Images were processed using Photoshop CS2 software (Adobe Systems). All images shown are representative of 3 to 5 independent experiments.

Flow cytometric analysis and cell sorting. Flow cytometry and isolation of blood cells from fetal liver and BM was performed by the following method. Briefly, fetal livers dissected from E14.5 embryos or BM cells obtained from femur and tibia were dispersed into single-cell suspension by gentle pipetting ${ }^{55}$. Fluorescencelabeled anti-CD34 (12-0349-42, eBioscience, dilution 1/100), -Flk2/CD135 (135309, dilution 1/100), -Sca-1 (108127, dilution 1/100), -c-Kit (105825, dilution 
Fig. 7 The effect of Regnase-1 on HSPCs is mediated through Gata2 and Tal1. a Relative expression of Gata2, Tal1, and Bmi1 in CD34- HSCs infected with shRNA virus targeting Gata2, Tal1, Bmil or scrambled control (shCtrl). Bmi1 was used as a non-candidate negative control. Data are expressed as foldchange relative to control shRNA infection ( $n=3$ per group). b Colony-forming assay of Regnase-1-deficient CD34- HSCs infected with either an shCtrl, shGata2, or shTal1 shRNA virus (left), and control (fl/fl) or Regnase-1-KO $(\Delta / \Delta)$ CD34- HSCs infected with control or shGata2+ shTal1 shRNA virus (right). G indicates CFU-granulocyte; GM CFU-granulocyte/monocyte; M CFU-monocyte; E CFU-erythroid; and mix mixed CFU-granulocytes, monocyte, erythroid, and megakaryocyte ( $n=4$ per group; 4 independent experiments). c Regnase-1-KO $(\Delta / \Delta)$ CD34- HSCs infected with shCtrl or shGata2+ shTal1 shRNA virus were transplanted and analyzed by flow cytometry ( $n=3$ per group; 3 independent experiments). $\mathbf{d}-\mathbf{f}$ Numbers and populations of LSK-HSCs or CD34- HSCs in total BM assessed by flow cytometry in $\mathbf{c}$. Data represent the means \pm SD. $\mathbf{g}$ Representative gross appearance of the spleen and mesenteric lymph nodes (MLNs) collected from bone marrow-transplanted mice as shown in $\mathbf{c}$. The scale bars represent $1 \mathrm{~cm}$. $\mathbf{h}$ Cell-cycle analysis of control BM CD34- HSCs in c by flow cytometry using EdU/7AAD staining. Dot plots indicate the frequency of $\mathrm{CD}^{-} 4^{-} \mathrm{HSCs}^{-}$in $\mathrm{GO}^{-\mathrm{G}}$ (EdU ${ }^{-} 7 \mathrm{AAD}^{-}$), $\mathrm{G} 2 / \mathrm{M}\left(\mathrm{EdU}^{+}\right)$, or $\mathrm{S}\left(\mathrm{EdU}^{+} 7 \mathrm{AAD}{ }^{+}\right)$phase of the cell cycle $\left(n=3\right.$ per group; 2 independent experiments). Error bars indicate mean $\pm \mathrm{SD} .{ }^{\star} p<0.05 ;{ }^{\star \star} p<$ $0.01,{ }^{\star \star *} p<0.005$, two-sided $t$-test in $\mathbf{a}, \mathbf{d}, \mathbf{e}, \mathbf{g}, \mathbf{h}$. Regnase-1 is known to mediate post-trasncriptional regulatory activity through degradation of target mRNAs. Here, the authors show that Regnase-1 regulates self-renewal of haematopoietic stem and progenitor cells through modulation of the stability of Gata2 and Tal1 mRNA

1/100), -Fc $\gamma \mathrm{R}$ (101325, dilution 1/100), -IL7Ra (121111, dilution 1/100), -CD71 (113807, dilution 1/100), -CD3 (100205, dilution 1/100), -Mac1 (101205, dilution 1/100), -B220 (103205, dilution 1/100), -Gr1 (108407, dilution 1/100), -CD45 (103134, dilution 1/100), -CD93 (136503, dilution 1/100), -CD150 (115909, dilution 1/100), -CD48 (103403, dilution 1/100), -CD45.1 (110730, dilution 1/100), -lineage Cocktail antibodies (133301, all purchased from BioLegend, dilution 1/30) were used. Stained cells were sorted by FACS Aria (BD Biosciences) and analyzed with FlowJo software (TreeStar). Gating strategies are shown in each figure and Supplementary Fig. 8. For RNA expression analysis and RNA-seq experiments, mRNA was directly isolated from sorted cells. For Giemsa staining, sorted cells were spun down onto glass slides using a Cytospin 4 instrument (Thermo Scientific) and stained with May-Grünwald-Giemsa (Muto Pure Chemicals).

Quantitative real-time PCR (qRT-PCR). Target gene mRNA levels were quantified by qRT-PCR or Western blotting as described ${ }^{56}$. PCR primers used in this work are described in Supplementary Data 2.

Colony-forming cell (CFC) assay. CFC assays were performed by culturing LSK cells $\left(1 \times 10^{3} /\right.$ dish $)$ or CD $34^{-}$HSCs $\left(\right.$CD $34^{-}$Flk $2^{-}$LSK $)\left(1 \times 10^{3} /\right.$ dish $)$ collected from $\mathrm{BM}$ in triplicate $35-\mathrm{mm}$ petri dishes (FALCON) containing $1 \mathrm{ml}$ MethoCult GF M3434 medium (Stem Cell Technologies). After 10 days of incubation at $37^{\circ} \mathrm{C}$ in $5 \% \mathrm{CO}_{2}$ in air, colony forming unit-granulocyte monocyte (CFU-GM), colony forming unit-granulocyte (CFU-G), colony forming unit-monocyte (CFU-M), and colony-forming unit mix (CFU-Mix) were counted.

Cell-cycle analysis and apoptosis assay. The proportion of HSPCs in G0/G1, $\mathrm{G} 2 / \mathrm{M}$, and $\mathrm{S}$ phases was analyzed using EdU incorporation assay over $24 \mathrm{~h}$ using Click-it EdU Alexa Fluor 647 Cell Flow Cytometry Assay Kit and Click-iT Plus EdU Alexa Fluor 488 Flow Cytometry Assay Kit (Thermo Fisher Scientific) according to the manufacturer's instructions. DNA content was detected with $7-$ AAD. To distinguish between cells in G0 and G1 phases, BM cells were stained with Hoechst 33342 at $37^{\circ} \mathrm{C}$. After $45 \mathrm{~min}, 1 \mu \mathrm{g} / \mathrm{ml} \mathrm{PY}$ was added and cells were incubated at $37^{\circ} \mathrm{C}$ for $45 \mathrm{~min}^{57}$. Samples were analyzed by FACSAria flow cytometry. Apoptotic cells were analyzed using the APC-Annexin V/Dead Cell Apoptosis Kit (Invitrogen) according to the manufacturer's protocol. Briefly, BM cells were treated with reagents provided in the kit and analyzed by FACS Aria flow cytometry and quantified using the FlowJo software.

Competitive transplantation assay. CD $34^{-}$HSCs (500 cells) from Reg1 1 flox/flox or Reg1 $1^{\Delta / \Delta}$ mice were mixed with wild-type CD $45.1^{+}$mice BM cells $\left(5 \times 10^{5}\right)$ and transplanted intravenously into lethally irradiated $(8 \mathrm{~Gy}) \mathrm{CD} 45.1^{+}$recipient mice. Sixteen weeks after transplantation, we assessed chimerism of donor-derived HSPCs and peripheral blood (PB) in recipient mice.

Myelosuppression models. Mice were intraperitoneally administered 5fluorouracil (5-FU; Kyowa Hakko) at a dose of $250 \mathrm{mg} / \mathrm{kg}$ body weight once per week for 2 weeks and their survival was monitored daily in the latter week. Bone marrow cells were collected and analyzed after $0,2,4,5$, and 6 days of administration.

Hemoglobin measurement. Hemoglobin concentration was quantified using a Hemoglobin Assay Kit (Biochain) according to the manufacturer's instructions.

Northern blotting. Total cellular RNA was prepared from THP1 cells transfected with a Regnase-1 expression plasmid or a control plasmid using RNeasy mini kits (Qiagen). RNAs were resolved by electrophoresis on a formaldehyde/agarose gel and transferred onto a positively charged nylon membrane (GE Healthcare). Probe preparation and hybridization were conducted with the DIG Northern Starter Kit (Roche Diagnostics), according to the manufacturer's protocol. DIG-labeled RNA probes targeting the Gata2 and Tal1 gene were constructed by T7 RNA polymerase reaction using the primer sets described in Supplementary Data 2. The human $\beta$ actin RNA probe was used to normalize the amount of RNA bound to the membrane.

Stability of mRNA. CD34- HSCs sorted from control (fl/fl) or Regnase-1-KO $(\Delta / \Delta)$ mouse BM were cultured for $1 \mathrm{~h}$. Actinomycin D (SIGMA) at $5 \mu \mathrm{g} / \mathrm{ml}$ was added to the culture medium to terminate transcription, and total RNA was harvested at the indicated time ${ }^{16}$. RNA was subjected to qRT-PCR to determine Gata2, Tal1, and CD34 levels.

Chromatin immunoprecipitation (ChIP) assay. The ChIP assay was performed using the SimpleChIP Plus Enzymatic Chromatin IP Kit (Cell Signaling Technology), according to the manufacturer's protocol. Chromatin solutions were prepared from THP1 cells transfected with a Regnasel expression plasmid or a control plasmid, digested with micrococcal nuclease and sonicated to give the desired fragment length. Immunoprecipitation was performed overnight at $4{ }^{\circ} \mathrm{C}$ using an anti-Gata2 antibody (ab22849, Abcam, dilution 1/200) or anti-Tal1 antibody (C15200012, Diagenode, dilution 1/200). PCR analysis was then performed using the primer sets described in Supplementary Data 2. The specificity of the assay was validated by normal IgG as the negative control.

Luciferase assay. Validation of Regnase-1 target genes by luciferase assay was performed as described ${ }^{33}$. Fragments of the $3^{\prime}$ UTR of the mRNA of interest were amplified with PCR using a specific primer (Supplementary Data 2) and inserted into the pGL3-luciferase plasmid. HEK293 cells were co-transfected with pGL3-3 UTR plasmid or pGL3-empty plasmid together with Regnase-1 expression plasmid or empty control plasmid. After $48 \mathrm{~h}$, cells were lysed and luciferase activity determined using the Dual-Luciferase Reporter Assay System (Promega). Renilla luciferase was transfected simultaneously and served as an internal control.

Retroviral vector construction and transduction. For knockdown experiments, oligonucleotides designed to target Gata2, Tal1, or Bmil (described in Supplementary Data 2) and scrambled oligonucleotides supplied by the manufacturer were annealed and cloned into the RNAi-ready pSIREN-RetroQ-zsGreen vector (Takara). Retroviruses were produced using PlatE packaging cells transfected with different plasmids as previously described ${ }^{58}$. Retrovirus supernatants were harvested 48 post-transfection and concentrated using the Retro-X Concentrator (Clontech). For transduction, $5 \times 10^{4} \mathrm{CD} 34^{-}$HSCs sorted by flow cytometry were incubated for $72 \mathrm{~h}$ in cytokine conditioned medium ( $1 \% \mathrm{FCS}, 100 \mathrm{ng} / \mathrm{mL}$ TPO, $100 \mathrm{ng} / \mathrm{mL}$ SCF, and $0.4 \mathrm{ng} / \mathrm{ml}$ IL-3) supplemented with virus particles. After transduction, cells were collected and used for CFC assays or bone marrow transplantation.

cDNA libraries and RNA sequencing. RNA samples isolated from CD34- $\mathrm{HSCs}$ from control Reg1 $1^{\text {flox/flox }}$ and $\operatorname{Reg} 1^{\Delta / \Delta}$ (Vav1-iCre; Regnase ${ }^{-} 1^{\text {flox/flox }}$ ) mice were reverse transcribed and amplified using the SMARTer Ultra Low Input RNA Kit for Sequencing-v3 (Takara Bio. Cat. 634849). Illumina sequencing libraries were constructed using Nextera XT DNA Librart Prep Kit (Illumina). After evaluating the quality and quantity of the constructed RNA-Seq libraries using a BioAnalyzer (Agilent Technologies), sequencing was performed on the HiSeq2500 platform with a 101-base paired-end read. Generated RNA-Seq tags were mapped to the reference mouse genome (GRCm38.p4) using STAR v2.4.2a ${ }^{59}$. We used the GENCODE annotation file (release version M17) for the mouse which can be downloaded from http://www.gencodegenes.org/. The tag counts of the first and last exons including $5^{\prime}$-untranslated and $3^{\prime}$-untranslated regions for each transcript 
were calculated, and then normalized by the lengths of the exons. The transcripts per million (TPM) values for each transcript were also calculated with Salmon $\mathrm{v} 0.9 .1^{60}$.

Identification of key factors by bioinformatics analysis. The microarray data set GSE69760 from BM and fetal liver Lineage ${ }^{-} \mathrm{Sca}^{-1}{ }^{+} \mathrm{c}-\mathrm{Kit}^{+}$(LSK) HSPCs in developmental stages were downloaded from Gene Expression Omnibus. To identify the crucial factors in regulation of self-renewal and differentiation of HSPCs, we screened genes according to the following criteria: (1) Expression level in adult BM was $>2$. (2) Expression level in adult BM was $>10$ times that of HSPCs from E14.5 FL. (3) Gene encodes intercellular proteins. (4) Gene encodes a cytokine, enzyme, G-protein coupled receptor, growth factor, ion channel, kinase, ligand-dependent nuclear receptor, peptidase, phosphatase, transcription regulator, translation regulator, transmembrane receptor, or transporter which are categorized in the Ingenuity Knowledge Base (https://www.qiagenbioinformatics.com/ products/ingenuity-pathway-analysis). After filtering, 10 genes were selected in Fig. 1a.

Gene set analysis. By analyzing RNA-seq gene expression profiles of Vav1-iCre; Reg $1^{\text {flox/flox }}$ mice (Reg $1^{\Delta / \Delta}$, case) and 8-week-old control Reg $1^{\text {flox } / \text { flox }}$ mice (Regl $1^{\text {flox/flox }}$, control), differential expression analysis was performed with the Bioconductor R package limma ${ }^{61}$. A total of 801 up-regulated genes in the Reg1 ${ }^{\Delta / \Delta}$ animals was detected according to the following criteria: $\log 2$-fold change $>2 \times$ the average expression value in Reg1 ${ }^{\Delta / \Delta}$ (case) compared with Reg $1^{\text {flox/flox }}$ (control); average expression value in the case was $>90$ th percentile of the case, and the FDR value was $<10^{-3}$. Reciprocally, 1179 down-regulated genes in Reg $1^{\Delta / \Delta}$ animals were detected according to the following criteria: $\log 2$-fold change $<1 / 2$ of the average expression value in cases compared with controls; average expression value in the control was $>90$ th percentile of the control, and the FDR value was $<10^{-3}$. Supplementary Data 3 in this differential expression analysis. To identify gene sets enriched among these down-regulated genes we used the Metascape analyses from the web site http://metascape.org//62. The results are summarized in Supplementary Data 4 . We also performed gene set enrichment analysis (GSEA) for a ranked list of genes using $\log 2$-fold change ${ }^{63}$. Based on the GSE12662 data, we identified genes with high or low expression in AML (18 M1, $19 \mathrm{M} 2$, and $14 \mathrm{M} 3$ patients) relative to normal bone marrow cells $(n=5)$ by the following method: (a) upregulated genes with a case mean/control mean ratio $>2$, $t$-test $p$-value $<1 \mathrm{e}-3$, and case mean expression $\geq 75$ th percentile of mean expression; (b) down-regulated genes with a case mean/control mean ratio $<1 / 2, t$-test $p$-value $<1 \mathrm{e}-3$, and control mean expression $\geq 75$ th percentile of mean expression. Gene expression data of Regnase-1-deficient mice were correlated with orthologous human genes, and whether the AML signature genes were coordinately expressed in LT-HSCs from Regnase- 1 deficient mice was assessed by GSEA. For our analyses, we used our predefined gene sets which are related to leukemia (Supplementary Data 5) and we considered gene sets with FDR $<0.1$ and $p$-value $<0.05$ as significant. The results of the GSEA are summarized in Supplementary Data 6.

Survival analysis based on Regnase- 1 target gene expression. To construct the prognostic model for AML patients based on the expression profiles of 801 target genes directly or indirectly down-regulated by Regnase-1 categorized as hematopoietic genes in the Ingenuity Knowledge Base, we downloaded the microarray expression data set GSE12417 from the Gene Expression Omnibus (GEO) database. We then used a Lasso-regularized Cox proportional hazard model with the glmnet package (version 2.0-5) (ref. ${ }^{64}$ ) in the R statistical environment (version 3.3.2) to build a Regnase- 1 signature-based prognostic classifier. Among the 801 genes in mouse, 242 genes were mapped to that orthologous genes of human in this dataset by using the Ingenuity Knowledge Base. The tuning parameter in the Lasso regularization was chosen by cross-validation. Using the prognostic model, patients were classified into two groups based on whether the risk score in the Cox model was more than 0 (high risk and worse prognosis) or less than 0 (low risk and better prognosis). To evaluate the prognostic significance of the Cox model, we used the Kaplan-Meier method and the $p$-value was calculated using the log-rank test.

Statistical analysis. All data are presented as the means \pm s.d. None of the data were excluded from the analyses. Investigators were not blinded to allocation during cell and mouse assays. For in vivo studies, mice of the same age and similar weight were randomly assigned to experimental or control groups. For statistical analysis, the statcel 4 software package (OMS, Saitama, Japan) was used, with analysis of variance performed on all data, followed by the Tukey-Kramer multiple comparison test. When only two groups were compared, a two-sided Student's $t$-test was used.

Reporting summary. Further information on experimental design is available in the Nature Research Reporting Summary linked to this article.

\section{Data availability}

The authors declare that all data supporting the findings of this study are available within the article and its Supplementary information. Any other data are available from the authors upon reasonable request. The RNA-seq data used in this manuscript are available in the GEO under the accession code GSE125546.

Received: 6 September 2017 Accepted: 18 February 2019

Published online: 06 March 2019

\section{References}

1. Orford, K. W. \& Scadden, D. T. Deconstructing stem cell self-renewal: genetic insights into cell-cycle regulation. Nat. Rev. Genet. 9, 115-128 (2008).

2. Wilson, A. et al. Hematopoietic stem cells reversibly switch from dormancy to self-renewal during homeostasis and repair. Cell 135, 1118-1129 (2008).

3. Morrison, S. J. \& Scadden, D. T. The bone marrow niche for haematopoietic stem cells. Nature 505, 327-334 (2014).

4. Iwasaki, H. et al. The order of expression of transcription factors directs hierarchical specification of hematopoietic lineages. Genes Dev. 20, 3010-3021 (2006).

5. He, S., Nakada, D. \& Morrison, S. J. Mechanisms of stem cell self-renewal. Annu. Rev. Cell Dev. Biol. 25, 377-406 (2009).

6. Shlush, L. I. \& Minden, M. D. Preleukemia: the normal side of cancer. Curr. Opin. Hematol. 22, 77-84 (2015).

7. Kreso, A. \& Dick, J. E. Evolution of the cancer stem cell model. Cell Stem Cell 14, 275-291 (2014)

8. Shima, Y. \& Kitabayashi, I. Deregulated transcription factors in leukemia. Int. J. Hematol. 94, 134-141 (2011).

9. Conne, B., Stutz, A. \& Vassalli, J. D. The 3' untranslated region of messenger RNA: a molecular 'hotspot' for pathology? Nat. Med. 6, 637-641 (2000).

10. Mayr, C. Evolution and biological roles of alternative 3'UTRs. Trends Cell Biol. 26, 227-237 (2016).

11. Schwerk, J. \& Savan, R. Translating the untranslated region. J. Immunol. 195 2963-2971 (2015)

12. Vlasova-St Louis, I. \& Bohjanen, P. R. Feedback regulation of kinase signaling pathways by AREs and GREs. Cells 5, E4 (2016).

13. Mizgalska, D. et al. Interleukin-1-inducible MCPIP protein has structural and functional properties of RNase and participates in degradation of IL-1beta mRNA. Febs. J. 276, 7386-7399 (2009).

14. Matsushita, K. et al. Zc3h12a is an RNase essential for controlling immune responses by regulating mRNA decay. Nature 458, 1185-1190 (2009).

15. Li, M. et al. MCPIP1 down-regulates IL-2 expression through an AREindependent pathway. PLoS ONE 7, e49841 (2012).

16. Uehata, T. et al. Malt1-induced cleavage of regnase- 1 in CD4(+) helper T cells regulates immune activation. Cell 153, 1036-1049 (2013).

17. Mino, T. et al. Regnase- 1 and roquin regulate a common element in inflammatory mrnas by spatiotemporally distinct mechanisms. Cell 161 1058-1073 (2015)

18. Younce, C. \& Kolattukudy, P. MCP-1 induced protein promotes adipogenesis via oxidative stress, endoplasmic reticulum stress and autophagy. Cell. Physiol. Biochem. 30, 307-320 (2012).

19. Vrotsos, E. G., Kolattukudy, P. E. \& Sugaya, K. MCP-1 involvement in glial differentiation of neuroprogenitor cells through APP signaling. Brain Res. Bull. 79, 97-103 (2009).

20. Labedz-Maslowska, A. et al. Monocyte Chemoattractant Protein-Induced Protein 1 (MCPIP1) enhances angiogenic and cardiomyogenic potential of murine bone marrow-derived mesenchymal stem cells. PLoS ONE $\mathbf{1 0}$ e0133746 (2015).

21. Adolfsson, J. et al. Upregulation of Flt3 expression within the bone marrow $\operatorname{Lin}(-) \mathrm{Scal}(+) \mathrm{c}-\mathrm{kit}(+)$ stem cell compartment is accompanied by loss of selfrenewal capacity. Immunity 15, 659-669 (2001).

22. Christensen, J. L. \& Weissman, I. L. Flk-2 is a marker in hematopoietic stem cell differentiation: a simple method to isolate long-term stem cells. Proc. Natl Acad. Sci. USA 98, 14541-14546 (2001).

23. Osawa, M., Hanada, K., Hamada, H. \& Nakauchi, H. Long-term lymphohematopoietic reconstitution by a single CD34- low/negative hematopoietic stem cell. Science 273, 242-245 (1996).

24. Ogilvy, S. et al. Constitutive Bcl-2 expression throughout the hematopoietic compartment affects multiple lineages and enhances progenitor cell survival. Proc. Natl Acad. Sci. USA 96, 14943-14948 (1999).

25. Cumano, A. \& Godin, I. Ontogeny of the hematopoietic system. Annu. Rev. Immunol. 25, 745-785 (2007)

26. Oguro, H., Ding, L. \& Morrison, S. J. SLAM family markers resolve functionally distinct subpopulations of hematopoietic stem cells and multipotent progenitors. Cell Stem Cell 13, 102-116 (2013).

27. Venezia, T. A. et al. Molecular signatures of proliferation and quiescence in hematopoietic stem cells. PLoS Biol. 2, e301 (2004).

28. Wilson, A. et al. Dormant and self-renewing hematopoietic stem cells and their niches. Ann. N.Y. Acad. Sci. 1106, 64-75 (2007) 
29. Grassinger, J., Haylock, D. N., Williams, B., Olsen, G. H. \& Nilsson, S. K. Phenotypically identical hemopoietic stem cells isolated from different regions of bone marrow have different biologic potential. Blood 116, 3185-3196 (2010).

30. Grassinger, J., Williams, B., Olsen, G. H., Haylock, D. N. \& Nilsson, S. K. Granulocyte colony stimulating factor expands hematopoietic stem cells within the central but not endosteal bone marrow region. Cytokine $\mathbf{5 8}$ 218-225 (2012).

31. Akira, S. Regnase-1, a ribonuclease involved in the regulation of immune responses. Cold Spring Harb. Symp. Quant. Biol. 78, 51-60 (2013).

32. Jeltsch, K. M. \& Heissmeyer, V. Regulation of T cell signaling and autoimmunity by RNA-binding proteins. Curr. Opin. Immunol. 39, 127-135 (2016).

33. Iwasaki, H. et al. The IkappaB kinase complex regulates the stability of cytokine-encoding mRNA induced by TLR-IL-1R by controlling degradation of regnase-1. Nat. Immunol. 12, 1167-1175 (2011).

34. Gottgens, B. Regulatory network control of blood stem cells. Blood $\mathbf{1 2 5}$ 2614-2620 (2015).

35. Ebina, W. \& Rossi, D. J. Transcription factor-mediated reprogramming toward hematopoietic stem cells. EMBO J. 34, 694-709 (2015).

36. Wang, Z. \& Ema, H. Mechanisms of self-renewal in hematopoietic stem cells. Int. J. Hematol. 103, 498-509 (2016).

37. McKinney-Freeman, S. et al. The transcriptional landscape of hematopoietic stem cell ontogeny. Cell Stem Cell 11, 701-714 (2012).

38. Zhou, L. et al. Monocyte chemoattractant protein-1 induces a novel transcription factor that causes cardiac myocyte apoptosis and ventricular dysfunction. Circ. Res. 98, 1177-1185 (2006).

39. Suzuki, H. I. et al. MCPIP1 ribonuclease antagonizes dicer and terminates microRNA biogenesis through precursor microRNA degradation. Mol. Cell 44, 424-436 (2011).

40. Fu, M. \& Blackshear, P. J. RNA-binding proteins in immune regulation: a focus on CCCH zinc finger proteins. Nat. Rev. Immunol. 17, 130-143 (2017).

41. Behrens, G. et al. A translational silencing function of MCPIP1/Regnase1 specified by the target site context. Nucleic Acids Res. 46, 4256-4270 (2018).

42. Tsai, F. Y. et al. An early haematopoietic defect in mice lacking the transcription factor GATA-2. Nature 371, 221-226 (1994).

43. Kitajima, K. et al. Redirecting differentiation of hematopoietic progenitors by a transcription factor, GATA-2. Blood 107, 1857-1863 (2006).

44. Hahn, C. N. et al. Heritable GATA2 mutations associated with familial myelodysplastic syndrome and acute myeloid leukemia. Nat. Genet. 43 1012-1017 (2011).

45. Katsumura, K. R., Ong, I. M., DeVilbiss, A. W., Sanalkumar, R. \& Bresnick, E. H. GATA factor-dependent positive-feedback circuit in acute myeloid leukemia cells. Cell Rep. 16, 2428-2441 (2016).

46. Luesink, M. et al. High GATA2 expression is a poor prognostic marker in pediatric acute myeloid leukemia. Blood 120, 2064-2075 (2012).

47. Ferrando, A. A. et al. Gene expression signatures define novel oncogenic pathways in $\mathrm{T}$ cell acute lymphoblastic leukemia. Cancer Cell 1, 75-87 (2002).

48. Sanda, T. et al. Core transcriptional regulatory circuit controlled by the TAL1 complex in human $\mathrm{T}$ cell acute lymphoblastic leukemia. Cancer Cell 22, 209-221 (2012).

49. O’Neil, J., Shank, J., Cusson, N., Murre, C. \& Kelliher, M. TAL1/SCL induces leukemia by inhibiting the transcriptional activity of E47/HEB. Cancer Cell 5, 587-596 (2004).

50. Palomero, T. et al. Transcriptional regulatory networks downstream of TAL1/ SCL in T-cell acute lymphoblastic leukemia. Blood 108, 986-992 (2006).

51. Jeltsch, K. M. et al. Cleavage of roquin and regnase- 1 by the paracaspase MALT1 releases their cooperatively repressed targets to promote $\mathrm{T}(\mathrm{H}) 17$ differentiation. Nat. Immunol. 15, 1079-1089 (2014).

52. Li, H., He, H., Gong, L., Fu, M. \& Wang, T. T. Short communication: preferential killing of HIV latently infected CD4(+) T cells by MALT1 inhibitor. AIDS Res. Hum. Retrovir. 32, 174-177 (2016).

53. Fontan, L. et al. MALT1 small molecule inhibitors specifically suppress ABCDLBCL in vitro and in vivo. Cancer Cell 22, 812-824 (2012).

54. Kawamoto, T. Use of a new adhesive film for the preparation of multi-purpose fresh-frozen sections from hard tissues, whole-animals, insects and plants. Arch. Histol. Cytol. 66, 123-143 (2003).

55. Takakura, N. et al. A role for hematopoietic stem cells in promoting angiogenesis. Cell 102, 199-209 (2000).

56. Kidoya, $\mathrm{H}$. et al. APJ regulates parallel alignment of arteries and veins in the skin. Dev. Cell 33, 247-259 (2015).

57. Naito, H., Kidoya, H., Sakimoto, S., Wakabayashi, T. \& Takakura, N. Identification and characterization of a resident vascular stem/progenitor cell population in preexisting blood vessels. EMBO J. 31, 842-855 (2012)
58. Satoh, T. et al. Critical role of Trib1 in differentiation of tissue-resident M2like macrophages. Nature 495, 524-528 (2013).

59. Dobin, A. et al. STAR: ultrafast universal RNA-seq aligner. Bioinformatics 29, 15-21 (2013).

60. Patro, R., Duggal, G., Love, M. I., Irizarry, R. A. \& Kingsford, C. Salmon provides fast and bias-aware quantification of transcript expression. Nat. Methods 14, 417-419 (2017).

61. Ritchie, M. E. et al. limma powers differential expression analyses for RNAsequencing and microarray studies. Nucleic Acids Res. 43, e47 (2015).

62. Tripathi, S. et al. Meta- and orthogonal integration of Influenza "OMICs" data defines a role for UBR4 in virus budding. Cell. Host Microbe 18, 723-735 (2015).

63. Subramanian, A. et al. Gene set enrichment analysis: a knowledge-based approach for interpreting genome-wide expression profiles. Proc. Natl Acad. Sci. USA 102, 15545-15550 (2005).

64. Simon, M. S. et al. Prospective analysis of association between use of statins or other lipid-lowering agents and colorectal cancer risk. Ann. Epidemiol. 22, 17-27 (2012).

\section{Acknowledgements}

We thank Dr. O. Takeuchi (IFLMS, Kyoto University, Japan) for supplying mutant mice, Dr. D. Okuzaki (RIMD, Osaka University, Japan) for performing RNA-seq analysis and Mr. R. Sasaki (Microeyes, Japan) for his help with the microscopic analysis. We are also grateful to Ms. S. Urakami, Ms. M. Omiya, Ms. Y. Mori, and Ms. N. Fujimoto for technical assistance. This work was supported by Grant-in Aid for Young Scientist A (No. 16H06147), Grant-in-Aid for Challenging Exploratory Research (15K14380) from the Japan Society for the Promotion of Science (JSPS), Takeda Science Foundation, SGH Foundation, the Kanae Foundation for the Promotion of Medical Science, the Uehara Memorial Foundation, Inamori Foundation, Ichiro Kanehara Foundation, the Mochida Memorial Foundation for Medical and Pharmaceutical Research, the Princess Takamatsu Cancer Research Fund, Osaka Cancer Society Research Grant, the Daiichi-Sankyo Foundation of Life Science, the Nakajima Foundation, the Japan Foundation for Applied Enzymology, Japan Agency for Medical Research and Development (AMED) under Grant number (18cm0106508h0002, 18gm5010002s110) and Japan Society for the Promotion of Science (JSPS) Grants-in-Aid for Scientific Research (A) (16H02470).

\section{Author contributions}

H.K. and N.T. designed and performed most of the experiments. F.M., W.J., T.Sa., Y.H., H.N., Y.K., F.A., M.S., Y.S., T.O., and S.A. supervised assays and performed some experiments. T.Sh. analyzed and interpreted data. H.K. and N.T. analyzed results and wrote the manuscript.

\section{Additional information}

Supplementary Information accompanies this paper at https://doi.org/10.1038/s41467 019-09028-w.

Competing interests: The authors declare no competing interests.

Reprints and permission information is available online at http://npg.nature.com/ reprintsandpermissions/

Journal peer review information: Nature Communications thanks the anonymous reviewers for their contribution to the peer review of this work. Peer reviewer reports are available.

Publisher's note: Springer Nature remains neutral with regard to jurisdictional claims in published maps and institutional affiliations.

Open Access This article is licensed under a Creative Common Attribution 4.0 International License, which permits use, sharing, adaptation, distribution and reproduction in any medium or format, as long as you give appropriate credit to the original author(s) and the source, provide a link to the Creative Commons license, and indicate if changes were made. The images or other third party material in this article are included in the article's Creative Commons license, unless indicated otherwise in a credit line to the material. If material is not included in the article's Creative Commons license and your intended use is not permitted by statutory regulation or exceeds the permitted use, you will need to obtain permission directly from the copyright holder. To view a copy of this license, visit http://creativecommons.org/ licenses/by/4.0/

(C) The Author(s) 2019 